\title{
Z-AREA SALTSTONE DISPOSAL FACILITY GROUNDWATER MONITORING \\ REPORT (U)
}

\section{FOURTH QUARTER 1995 \\ AND 1995 SUMMARY}

Publication Date: March 1996

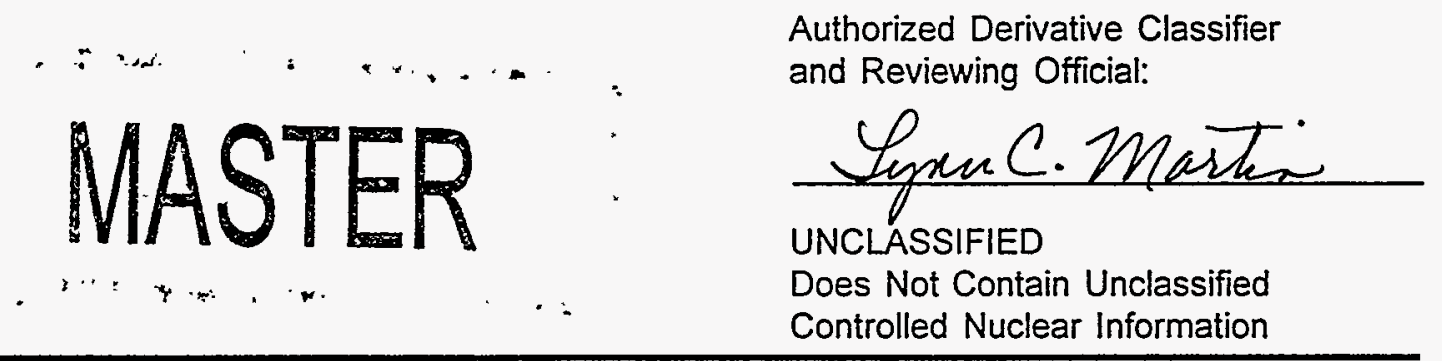

Westinghouse Savannah River Company Savannah River Site Aiken, SC 29808 


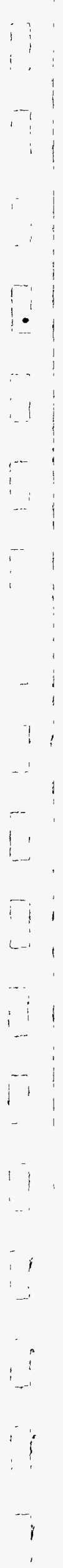


WSRC-TR-96-0054

Unclassified

\section{Z-AREA SALTSTONE DISPOSAL FACILITY GROUNDWATER MONITORING REPORT (U)}

FOURTH QUARTER 1995

AND 1995 SUMMARY

Publication Date: March 1996

Authorized Derivative Classifier and Reviewing Official:

UNCLASSIFIED

Does Not Contain Unclassified Controlled Nuclear Information

Westinghouse Savannah River Company

Savannah River Site

Aiken, SC 29808 


\section{DISCLAIMER}

This report was prepared as an account of work sponsored by an agency of the United States Government. Neither the United States Government nor any agency thereof, nor any of their employees, makes any warranty, express or implied, or assumes any legal liability or responsibility for the accuracy, completeness, or usefulness of any information, apparatus, product, or process disclosed, or represents that its use would not infringe privately owned rights. Reference herein to any specific commercial product, process, or service by trade name, trademark, manufacturer, or otherwise does not necessarily constitute or imply its endorsement, recommendation, or favoring by the United States Government or any agency thereof. The views and opinions of authors expressed herein do not necessarily state or reflect those of the United States Government or any agency thereof.

This report has been reproduced directly from the best available copy.

Available to DOE and DOE contractors from the Office of Scientific and Technical Information, P.O. Box 62, Oak Ridge, TN 37831; prices available from (615) 576-8401.

Available to the public from the National Technical Information Service, U.S. Department of Commerce, 5285 Port Royal Road, Springfield, VA 22161. 


\section{DISCLAIMER}

Portions of this document may be illegible in electronic image products. Images are produced from the best available original document. 


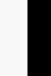


WSRC-TR-96-0054

Unclassified

\section{Z-AREA SALTSTONE DISPOSAL FACILITY GROUNDWATER MONITORING REPORT (U)}

\section{FOURTH QUARTER 1995 \\ AND 1995 SUMMARY}

Publication Date: March 1996

\section{Key Words}

dichloromethane

Industrial Waste Permit IWP-217

low-level radioactive waste

ZBG wells

Westinghouse Savannah River Company

Savannah River Site

Aiken, SC 29808 
WSRC-TR-96-0054

Unclassified

THIS PAGE LEFT BLANK INTENTIONALLY. 


\section{Abstract}

Samples from the ZBG wells at the Z-Area Saltstone Disposal Facility are analyzed quarterly for constituents required by South Carolina Department of Health and Environmental Control Industrial Waste Permit IWP-217 and for other constituents as part of the Savannah River Site (SRS) Groundwater Monitoring Program.

During fourth quarter 1995, no constituents were reported above final Primary Drinking Water Standards or SRS flagging criteria.

In the past, tritium has been detected sporadically in the ZBG wells at levels similar to those detected before $Z$ Area began radioactive operations. 
WSRC-TR-96-0054 Unclassified

THIS PAGE LEFT BLANK INTENTIONALLY. 


\section{Contents}

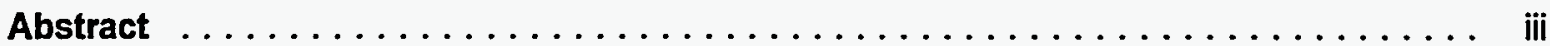

List of Figures $\ldots \ldots \ldots \ldots \ldots \ldots \ldots \ldots \ldots \ldots \ldots \ldots \ldots \ldots \ldots \ldots \ldots$

List of Tables $\ldots \ldots \ldots \ldots \ldots \ldots \ldots \ldots \ldots \ldots \ldots \ldots \ldots \ldots \ldots \ldots$ vi

Executive Summary $\ldots \ldots \ldots \ldots \ldots \ldots \ldots \ldots \ldots \ldots \ldots \ldots \ldots \ldots \ldots$



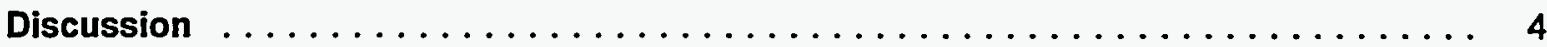

Groundwater Monitoring Data . . . . . . . . . . . . . . . . . . . . 4

Analytical Results Exceeding Standards $\ldots \ldots \ldots \ldots \ldots \ldots \ldots \ldots \ldots \ldots \ldots \ldots$

Upgradient vs. Downgradient Results $\ldots \ldots \ldots \ldots \ldots \ldots \ldots \ldots \ldots \ldots \ldots \ldots$

Conclusions $\ldots \ldots \ldots \ldots \ldots \ldots \ldots \ldots \ldots \ldots \ldots \ldots \ldots \ldots \ldots \ldots \ldots \ldots$



References Cited $\ldots \ldots \ldots \ldots \ldots \ldots \ldots \ldots \ldots \ldots \ldots \ldots \ldots \ldots \ldots \ldots$

Errata $\ldots \ldots \ldots \ldots \ldots \ldots \ldots \ldots \ldots \ldots \ldots \ldots \ldots \ldots \ldots \ldots \ldots$

Appendix A-Standards $\ldots \ldots \ldots \ldots \ldots \ldots \ldots \ldots \ldots \ldots \ldots \ldots \ldots \ldots \ldots$

Appendix B-Flagging Criteria $\ldots \ldots \ldots \ldots \ldots \ldots \ldots \ldots \ldots \ldots \ldots \ldots$

Appendix C-Figures $\ldots \ldots \ldots \ldots \ldots \ldots \ldots \ldots \ldots \ldots \ldots \ldots \ldots \ldots \ldots$

Appendix D-Groundwater Monitoring Results Tables $\ldots \ldots \ldots \ldots \ldots \ldots \ldots$

Appendix E-Data Quality/Usability Assessment $\ldots \ldots \ldots \ldots \ldots \ldots \ldots \ldots$ E-1 


\section{List of Figures}

Page

1. Location of the Z-Area Saltstone Disposal Facility at the Savannah River Site . . . . C-3

2. Location of the Z-Area Saltstone Disposal Facility within the Separations Area $\ldots .$. C-4

3. Location of Groundwater Monitoring Wells at the Z-Area Saltstone Disposal Facility . C C-5

\section{List of Tables}

Page

1. Maximum Levels of Constituents Exceeding the South Carolina Department of Health and Environmental Control Proposed Groundwater Monitoring Standards or the Final Primary Drinking Water Standards D-6

2. Maximum Levels of Constituents Exceeding Half the Final Primary Drinking Water Standards or Other SRS Flagging Criteria D-6

3. Groundwater Monitoring Results for Individual Wells D-7 


\section{Executive Summary}

The three monitoring wells at the Z-Area Saltstone Disposal Facility, ZBG 1, 1A, and 2, are sampled quarterly as part of the Savannah River Site (SRS) Groundwater Monitoring Program and to comply with conditions of the facility's Industrial Waste Permit IWP-217, issued by the South Carolina Department of Health and Environmental Control (SCDHEC). During fourth quarter 1995, samples from wells ZBG 1 and 2 were analyzed for selected inorganic constituents, volatile organic compounds, radionuclides, and other constituents. Well ZBG $1 \mathrm{~A}$ was dry and could not be sampled.

None of the ZBG wells contained constituents above final Primary Drinking Water Standards, SCDHEC proposed groundwater monitoring standards; or SRS flagging criteria during fourth quarter 1995.

In previous quarters, wells ZBG 1 and 2 contained slightly elevated levels of tritium, similar to the tritium levels detected before $Z$ Area began radioactive operations. 
WSRC-TR-96-0054

Unclassified

THIS PAGE LEFT BLANK INTENTIONALLY. 


\section{Introduction}

The Z-Area Saltstone Disposal Facility is located in the Separations Area, north of $\mathrm{H}$ and $\mathrm{S}$ Areas, at the Savannah River Site (SRS) (Figures 1 and 2, Appendix C). The facility permanently disposes of low-level radioactive waste in accordance with South Carolina Department of Health and Environmental Control (SCDHEC) Industrial Waste Permit IWP-217 (SCDHEC, 1989). The facility blends low-level radioactive salt solution with cement, slag, and flyash to form a nonhazardous cementitious waste that is pumped to aboveground disposal vaults. $Z$ Area began these operations in June 1990.

The site is monitored by three groundwater monitoring wells (Figure 3 , Appendix $C$ ). Wells ZBG 1 and 2 monitor the water table, while well ZBG $1 \mathrm{~A}$ monitors a zone where perched water has been observed in the past. Based on piezometric data for $Z$ Area obtained prior to application for the permit (DOE, 1989), wells ZBG 1 and $1 A$ are located upgradient of the disposal vaults, and well ZBG 2 is located downgradient of the vaults (Figure 3, Appendix C). 
WSRC-TR-96-0054

Unclassified

\section{Discussion}

\section{Groundwater Monitoring Data}

The Environmental Protection Department/Environmental Monitoring Section (EPD/EMS) collects quarterly groundwater samples from wells at the Z-Area Saltstone Disposal Facility as part of the SRS Groundwater Monitoring Program and to satisfy the requirements of the facility's waste permit. The sampling procedure (WSRC, 1992) requires evacuation of a minimum of two well volumes and stabilization of $\mathrm{pH}$, specific conductance, and turbidity prior to sample collection. Stability is established when three successive measurements, taken within a given time period, are within a specified tolerance range.

If a well pumps dry before two well volumes are purged or before stabilization is achieved, it must be revisited within 24 hours for the data generated to be considered the result of a single sampling event. On the second visit within 24 hours, samples are taken without further purging or stability measurements; thus, these samples may not be representative of groundwater quality.

During fourth quarter 1995, groundwater samples were analyzed for constituents required by SCDHEC Industrial Waste Permit IWP-217, including inorganic constituents, selected radionuclides, volatile organic compounds, and other constituents. Wells ZBG 1 and 2 have singlespeed centrifugal downhole pumps, and well ZBG $1 A$ is sampled with an open-bucket bailer.

SCDHEC-proposed groundwater monitoring standards, presented in permit IWP-217 (Table 1, Appendix A); Safe Drinking Water Act final Primary Drinking Water Standards (PDWS) or drinking water screening levels, established by the U.S. Environmental Protection Agency (EPA) (Table 2, Appendix A); the South Carolina final PDWS for lead (Table 2, Appendix A); or SRS flagging criteria (Appendix B) were used as standards for comparison. Monitoring results were compared either to the SCDHEC-proposed groundwater monitoring standards or to the final PDWS (depending on which was lower). The SRS flagging criteria are based on final and proposed PDWS, Secondary Drinking Water Standards, and method detection limits. Constituent levels that equal or exceed the SCDHEC standards, final PDWS, screening levels, or Flag 2 criteria are described as exceeding standards, or as elevated, and constituent levels that equal or exceed Flag 1 criteria (but not Flag 2 levels) are described as exceeding Flag 1 or as slightly elevated.

The final PDWS for individual analytes given in Appendix A may not always match the SRS flagging criteria in Appendix B. The final PDWS are used as guidelines in this compliance report to meet regulatory requirements; the flagging criteria are used by EPD/EMS to identify relative levels of constituents in the groundwater and as guides for scheduling groundwater sampling.

\section{Analytical Results Exceeding Standards}

No constituents exceeded final PDWS or SCDHEC proposed groundwater monitoring standards at the Z-Area Saltstone Disposal Facility during fourth quarter 1995 (Table 1, Appendix D). During second quarter 1995, dichloromethane, a common laboratory contaminant, exceeded the final PDWS in well ZBG 1 and was detected in this well's associated method blank. During fourth quarter 1995, dichloromethane was analyzed but was not above standards. 
Table 2 (Appendix D) shows that no other constituents exceeded the SRS Flag 1 or 2 criteria during fourth quarter 1995 in any ZBG well.

Table 3 (Appendix D) shows all the results for individual wells. Well ZBG 1A was dry and could not be sampled. Those results that received modifiers (which help identify laboratory accuracy and precision), exceeded either the SCDHEC standards or the final PDWS (whichever is lower), or exceeded the EPA-approved holding times during fourth quarter 1995 are noted in this table. Table 3 (Appendix D) also lists the number of well volumes purged from each ZBG well during fourth quarter 1995.

\section{Upgradient vs. Downgradient Results}

Wells ZBG 1 and $1 A$ are the designated upgradient wells at the Z-Area Saltstone Disposal Facility. During fourth quarter 1995, no constituents exceeded final PDWS in upgradient well ZBG 1 or downgradient well ZBG 2. Well ZBG $1 A$ was dry and could not be sampled. 


\section{Conclusions}

No constituents exceeded final PDWS or SCDHEC proposed groundwater monitoring standards at the Z-Area Saltstone Disposal Facility during fourth quarter 1995. Dichloromethane, a common laboratory contaminant that exceeded its final PDW/S in upgradient well ZBG 1 during second quarter 1995, was not elevated in any well during fourth quarter 1995.

Tritium, which has been slightly elevated in the past, did not exceed standards in any ZBG well sampled during first, second, third, or fourth quarter 1995. The tritium levels detected previously are similar to levels detected before $Z$ Area began radioactive operations on June 12, 1990. The source of the tritium in the Z-Area wells, therefore, is not from activities at the waste disposal facility.

SRS currently is in the process of renewing the Z-Area Saltstone Disposal Facility's Industrial Waste Permit IWP-217. 


\section{Summary}

During 1995, dichloromethane, which is a common laboratory contaminant, exceeded the final PDWS (5 ug/L) only in well ZBG 1 during second quarter. In 1994, dichloromethane exceeded the final PDWS only in well ZBG 2 during first quarter. Because dichloromethane occurs only sporadically, the elevated values probably are the result of laboratory contamination.

In 1994, chloromethane exceeded Flag 2 criteria in well ZBG 2 during third quarter. This constituent was not reported above standards during 1995.

Tritium, which exceeded the Flag 1 criterion in downgradient well ZBG 2 during first, second, and fourth quarter 1994, did not exceed standards in any ZBG well sampled during first, second, third, or fourth quarter 1995. The tritium levels detected previously are similar to levels detected before $Z$ Area began radioactive operations on June 12, 1990. The source of the tritium in the Z-Area wells, therefore, is not from activities at the waste disposal facility. 
WSRC-TR-96-0054

Unclassified

THIS PAGE LEFT BLANK INTENTIONALLY. 
WSRC-TR-96-0054

Unclassified

\section{Appendix A}

\section{Standards}


WSRC-TR-96-0054

Unclassified

THIS PAGE LEFT BLANK INTENTIONALLY. 
Table 1. South Carolina Department of Health and Environmental Control Proposed Groundwater Monitoring Standards (SCDHEC, 1989)

Analyte

Antimony

Arsenic

Barium

Cadmium

Chromium

Lead

Mercury

Nitrate as nitrogen

Nitrite as nitrogen

Selenium

Silver
Unit

$\mu g / L$

$\mu g / L$

$\mu g / L$

$\mu g / L$

$\mu g / L$

$\mu g / L$

$\mu \mathrm{g} / \mathrm{L}$

$\mu g / L$

$\mu g / L$

$\mu g / L$

$\mu \mathrm{g} / \mathrm{L}$
Standard

50

50

1,000

10

50

10

2

10,000

1,000

10

50

Note: Benzene and toluene concentrations are not to exceed concentrations or amounts that interfere with use, actual or intended, as determined by SCDHEC. Proposed standards for radionuclides at SRS are under jurisdictional assessment; therefore, no radionuclide standards are proposed for groundwater quality monitoring for the current permit application. 
Table 2. Final Primary Drinking Water Standards



\begin{tabular}{|c|c|c|c|}
\hline Unit & Level & Status & Source \\
\hline$\mu g / L$ & 2 & Final & EPA, 1993 \\
\hline$\mu g / L$ & 3 & Final & EPA, 1993 \\
\hline$\mu g / L$ & 2 & Final & EPA, 1993 \\
\hline$\mu g / L$ & 4 & Final & EPA, 1993 \\
\hline$\mu g / L$ & 6 & Final & EPA, 1993 \\
\hline$\mu g / L$ & 50 & Final & EPA, 1993 \\
\hline Fibers/L & $7,000,000$ & Final & EPA, 1993 \\
\hline$\mu g / L$ & 3 & Final & EPA, 1993 \\
\hline$\mu \mathrm{gg} / \mathrm{L}$ & 2,000 & Final & EPA, 1993 \\
\hline$\mu g / L$ & 5 & Final & EPA, 1993 \\
\hline$\mu g / L$ & 0.2 & Final & EPA, 1993 \\
\hline$\mu g / L$ & 4 & Final & EPA, 1993 \\
\hline$\mu g / L$ & 6 & Final & EPA, 1993 \\
\hline$\mu g / L$ & 100 & Final & EPA, 1993 \\
\hline$\mu g / L$ & 100 & Final & EPA, 1993 \\
\hline$\mu g / L$ & 7 & Final & EPA, 1993 \\
\hline$\mu g / L$ & 5 & Final & EPA, 1993 \\
\hline$\mu g / L$ & 40 & Final & EPA, 1993 \\
\hline$\mu g / L$ & 5 & Final & EPA, 1993 \\
\hline$\mu \mathrm{g} / \mathrm{L}$ & 2 & Final & EPA, 1993 \\
\hline$\mu g / L$ & 100 & Final & EPA, 1993 \\
\hline$\mu \mathrm{g} / \mathrm{L}$ & 2 & Final & EPA, 1993 \\
\hline$\mu \mathrm{g} / \mathrm{L}$ & 100 & Final & EPA, 1993 \\
\hline$\mu g / L$ & 100 & Final & EPA, 1993 \\
\hline$\mu g / L$ & 1,300 & Final & EPA, 1993 \\
\hline$\mu g / L$ & 200 & Final & EPA, 1993 \\
\hline$\mu g / L$ & 200 & Final & EPA, 1993 \\
\hline$\mu g / L$ & 100 & Final & EPA, 1993 \\
\hline$\mu g / L$ & 0.2 & Final & EPA, 1993 \\
\hline$\mu g / L$ & 0.05 & Final & EPA, 1993 \\
\hline$\mu g / L$ & 600 & Final & EPA, 1993 \\
\hline$\mu g / L$ & 75 & Final & EPA. 1993 \\
\hline$\mu g / L$ & 5 & Final & EPA, 1993 \\
\hline$\mu g / L$ & 7 & Final & EPA. 1993 \\
\hline$\mu g / L$ & 50 & Final & EPA. 1993 \\
\hline$\mu \mathrm{g} / \mathrm{L}$ & 70 & Final & EPA, 1993 \\
\hline$\mu g / L$ & 100 & Final & EPA, 1993 \\
\hline$\mu \mathrm{g} / \mathrm{L}$ & 5 & Final & EPA, 1993 \\
\hline$\mu g / L$ & 70 & Final & EPA, 1993 \\
\hline$\mu g / L$ & 5 & Final & EPA. 1993 \\
\hline$\mu g / L$ & 400 & Final & EPA, 1993 \\
\hline$\mu g / L$ & 20 & Final & EPA, 1993 \\
\hline$\mu g / L$ & 100 & Final & EPA, 1993 \\
\hline$\mu g / L$ & 2 & Final & EPA, 1993 \\
\hline$\mu g / L$ & 700 & Final & EPA, 1993 \\
\hline$\mu g / L$ & 4,000 & Final & EPA, 1993 \\
\hline$\mu g / L$ & 700 & Final & EPA, 1993 \\
\hline $\mathrm{pCi} / \mathrm{L}$ & $1.5 \mathrm{E}+01$ & Final & EPA, 1993 \\
\hline$\mu g / L$ & 0.4 & Final & EPA, 1993 \\
\hline$\mu \mathrm{g} / \mathrm{L}$ & 0.2 & Final & EPA, 1993 \\
\hline$\mu g / L$ & 1 & Final & EPA, 1993 \\
\hline$\mu g / L$ & 50 & Final & EPA, 1993 \\
\hline$/ L$ & 50 & Final & SCDHEC, 1 \\
\hline
\end{tabular}




\begin{tabular}{|c|c|c|c|c|}
\hline Analyte & Unit & Level & $\underline{\text { Status }}$ & Source \\
\hline Lindane & $\mu \mathrm{g} / \mathrm{L}$ & 0.2 & Final & EPA, 1993 \\
\hline Mercury & $\mu \mathrm{g} / \mathrm{L}$ & 2 & Final & EPA, 1993 \\
\hline Methoxychlor & $\mu \mathrm{g} / \mathrm{L}$ & 40 & Final & EPA, 1993 \\
\hline Nickel & $\mu g / L$ & 100 & Final & EPA, 1993 \\
\hline Nitrate as nitrogen & $\mu \mathrm{g} / \mathrm{L}$ & 10,000 & Final & EPA, 1993 \\
\hline Nitrate-nitrite as nitrogen & $\mu \mathrm{g} / \mathrm{L}$ & 10,000 & Final & EPA, 1993 \\
\hline Nitrite as nitrogen & $\mu \mathrm{g} / \mathrm{L}$ & 1,000 & Final & EPA, 1993 \\
\hline Nonvolatile beta & pCill & $5 \mathrm{E}+01$ & Interim Final & EPA, 1977 \\
\hline Oxamy $\left.\right|^{\mathrm{a}}$ & $\mu g / L$ & 200 & Final & EPA, 1993 \\
\hline PCB 1016 & $\mu \mathrm{g} / \mathrm{L}$ & 0.5 & Final & EPA, 1993 \\
\hline РCB 1221 & $\mu g / L$ & 0.5 & Final & EPA, 1993 \\
\hline PCB 1232 & $\mu \mathrm{g} / \mathrm{L}$ & 0.5 & Final & EPA, 1993 \\
\hline РCB 1242 & $\mu \mathrm{g} / \mathrm{L}$ & 0.5 & Final & EPA, 1993 \\
\hline РCB 1248 & $\mu g / L$ & 0.5 & Final & EPA, 1993 \\
\hline PCB 1254 & $\mu \mathrm{g} / \mathrm{L}$ & 0.5 & Final & EPA, 1993 \\
\hline PCB 1260 & $\mu \mathrm{g} / \mathrm{L}$ & 0.5 & Final & EPA, 1993 \\
\hline РCB 1262 & $\mu g / L$ & 0.5 & Final & EPA, 1993 \\
\hline Pentachlorophenol & $\mu \mathrm{g} / \mathrm{L}$ & 1 & Final & EPA, 1993 \\
\hline Picloram ${ }^{\mathrm{a}}$ & $\mu \mathrm{g} / \mathrm{L}$ & 500 & Final & EPA, 1993 \\
\hline Selenium & $\mu \mathrm{g} / \mathrm{L}$ & 50 & Final & EPA, 1993 \\
\hline Simazine $^{a}$ & $\mu \mathrm{g} / \mathrm{L}$ & 4 & Final & EPA, 1993 \\
\hline  & $\mathrm{pCi} / \mathrm{L}$ & $8 \mathrm{E}+00$ & Final & EPA, 1993 \\
\hline Strontium-90 & pCill & $8 E+00$ & Final & EPA, 1993 \\
\hline Styrene & $\mu g / L$ & 100 & Final & EPA, 1993 \\
\hline $2,3,7,8-T C D D$ & $\mu g / L$ & 0.00003 & Final & EPA, 1993 \\
\hline Tetrachloroethylene & $\mu \mathrm{g} / \mathrm{L}$ & 5 & Final & EPA, 1993 \\
\hline Thallium & $\mu \mathrm{g} / \mathrm{L}$ & 2 & Final & EPA, 1993 \\
\hline Toluene & $\mu \mathrm{g} / \mathrm{L}$ & 1,000 & Final & EPA, 1993 \\
\hline Toxaphene & $\mu \mathrm{g} / \mathrm{L}$ & 3 & Final & EPA, 1993 \\
\hline 2,4,5-TP (Silvex) & $\mu g / L$ & 50 & Final & EPA, 1993 \\
\hline 1,2,4-Trichlorobenzene & $\mu \mathrm{g} / \mathrm{L}$ & 70 & Final & EPA, 1993 \\
\hline 1,1,1-Trichloroethane & $\mu g / L$ & 200 & Final & EPA, 1993 \\
\hline 1,1,2-Trichloroethane & $\mu g / L$ & 5 & Final & EPA, 1993 \\
\hline Trichloroethylene & $\mu \mathrm{g} / \mathrm{L}$ & 5 & Final & EPA, 1993 \\
\hline Tritium & $\mathrm{pCi} / \mathrm{mL}$ & $2 E+01$ & Final & EPA, 1993 \\
\hline Xylenes & $\mu g / L$ & 10,000 & Final & EPA, 1993 \\
\hline
\end{tabular}

a At present, EMS does not perform this analysis because the constituent is not in the current contract.

b The standard given is for gross alpha including radium-226 but excluding radon and uranium.

c For double radionuclide analyses where each separate radionuclide has its own standard, the more stringent standard is used. 


\section{References Cited}

EPA (U.S. Environmental Protection Agency), 1977. National Interim Primary Drinking Water Regulations, EPA-570/9-76-003. Washington, DC.

EPA (U.S. Environmental Protection Agency), 1993. National Primary Drinking Water Regulations, Code of Federal Regulations, Title 40, Part 141, pp. 592-732. Washington, DC.

SCDHEC (South Carolina Department of Health and Environmental Control), 1981. State Primary Drinking Water Regulations, R.61-58.5. Columbia, SC.

SCDHEC (South Carolina Department of Health and Environmental Control), 1989. Industrial Solid Waste Permit Application for the Proposed Z-Area Saltstone Disposal Facility, Permit 12,683. Columbia, SC. 


\title{
Appendix B
}

\author{
Flagging Criteria
}


WSRC-TR-96-0054

Unclassifjed

THIS PAGE LEFT BLANK INTENTIONALLY. 


\section{Flagging Criteria}

The Savannah River Site Environmental Protection Department/Environmental Monitoring Section (EPD/EMS) flagging criteria are as follows:

- Flag 2 criteria for constituents equal the Safe Drinking Water Act (SDWA) final Primary Drinking Water Standards (PDWS), the SDWA proposed PDWS, or the SDWA Secondary Drinking Water Standards (SDWS). If a constituent does not have a drinking water standard, the Flag 2 criterion equals 10 times the method detection limit (MDL) calculated as the 90th percentile detection limit obtained recently by one of the primary analytical laboratories.

- Flag 1 criteria for constituents equal one-half of the final PDWS, one-half the proposed PDWS, or one-half the SDWS. If a constituent does not have a drinking water standard, the Flag 1 criterion equals 5 times the MDL calculated as the 90th percentile detection limit obtained recently by one of the primary analytical laboratories.

- Flag 0 criteria are assigned to constituent levels below Flag 1 criteria, constituent levels below the sample detection limits, or constituents having no flagging criteria.

The following parameters are exceptions to the flagging rules:

- EPD/EMS sets flagging criteria for $\mathrm{pH}$ and specific conductance. No flags are set for alkalinity, calcium, carbonate, magnesium, potassium, silica, sodium, total dissolved solids, total phosphates (as P), and total phosphorus. Analyses for these parameters are conducted as part of the biennial comprehensive analyses or by special request.

- Aesthetic parameters such as color, corrosivity, Eh, odor, surfactants, and turbidity are not assigned flagging criteria but are analyzed by special request.

- Common laboratory contaminants and cleaners such as dichloromethane (methylene chloride), ketones, phthalates, and toluene are not assigned flagging criteria unless they have PDWS. These constituents are analyzed by special request.

\begin{tabular}{|c|c|c|c|}
\hline Analyte & $\underline{\text { Unit }}$ & Flag 1 & Flag 2 \\
\hline Acenaphthene & $\mu \mathrm{g} / \mathrm{L}$ & 50 & 100 \\
\hline Acenaphthylene & $\mu \mathrm{g} / \mathrm{L}$ & 50 & 100 \\
\hline Acetone & $\mu \mathrm{g} / \mathrm{L}$ & 500 & 1,000 \\
\hline Acetonitrile (Methyl cyanide) & $\mu g / L$ & 500 & 1,000 \\
\hline Acetophenone & $\mu \mathrm{g} / \mathrm{L}$ & 50 & 100 \\
\hline 2-Acetylaminofluorene & $\mu \mathrm{g} / \mathrm{L}$ & 50 & 100 \\
\hline Acrolein & $\mu \mathrm{g} / \mathrm{L}$ & 100 & 200 \\
\hline Acrylonitrile & $\mu \mathrm{g} / \mathrm{L}$ & 100 & 200 \\
\hline Actinium-228 & $\mathrm{pCi} / \mathrm{L}$ & $1.64 \mathrm{E}+03$ & $3.27 E+03$ \\
\hline Alachlor & $\mu \mathrm{g} / \mathrm{L}$ & 1 & 2 \\
\hline Aldicarb $b^{b}$ & $\mu \mathrm{g} / \mathrm{L}$ & 1.5 & 3 \\
\hline Aldicarb sulfone $e^{b}$ & $\mu \mathrm{g} / \mathrm{L}$ & 1 & 2 \\
\hline Aldicarb sulfoxide ${ }^{b}$ & $\mu \mathrm{g} / \mathrm{L}$ & 2 & 4 \\
\hline Aldrin & $\mu \mathrm{g} / \mathrm{L}$ & 0.25 & 0.5 \\
\hline Alkalinity (as $\mathrm{CaCO}_{3}$ ) & & No flag & No flag \\
\hline Allyl chloride & $\mu g / L$ & 250 & 500 \\
\hline Aluminum & $\mu \mathrm{g} / \mathrm{L}$ & 25 & 50 \\
\hline Aluminum, dissolved & $\mu \mathrm{g} / \mathrm{L}$ & 25 & 50 \\
\hline Aluminum, total recoverable & $\mu \mathrm{g} / \mathrm{L}$ & 25 & 50 \\
\hline
\end{tabular}

Source $^{a}$
EPA Method 8270
EPA Method 8270
EPA Method 8240
EPA Method 8240
EPA Method 8270
EPA Method 8270
EPA Method 8240
EPA Method 8240
Proposed PDWS (EPA, 1991)
Final PDWS (EPA, 1993a)
Final PDWS (EPA, 1993a)
Final PDWS (EPA, 1993a)
Final PDWS (EPA, 1993a)
EPA Method 8080
Set by EPD/EMS
EPA Method 8240
SDWS (EPA, 1993b)
SDWS (EPA, 1993b)
SDWS (EPA, 1993b)




\begin{tabular}{|c|c|c|c|c|}
\hline Analyte & Unit & Flag 1 & Flag 2 & Source $^{a}$ \\
\hline Americium-241 & pCi/L & 3.17E+00 & $6.34 E+100$ & Proposed PDWS (EPA, 1991) \\
\hline Americium-243 & $\mathrm{pCi} / \mathrm{L}$ & $3.19 E+00$ & $6.37 E+100$ & Proposed PDWS (EPA, 1991) \\
\hline 4-Aminobiphenyl & $\mu g / L$ & 50 & 100 & EPA Method 8270 \\
\hline Ammonia & $\mu g / L$ & 500 & 1,000 & APHA Method 417B \\
\hline Ammonia nitrogen & $\mu g / L$ & 500 & 1,000 & EPA Method 350.1 \\
\hline Aniline & $\mu g / L$ & 50 & 100 & EPA Method 8270 \\
\hline Anthracene & $\mu g / L$ & 50 & 100 & EPA Method 8270 \\
\hline Antimony & $\mu \mathrm{g} / \mathrm{L}$ & 3 & 6 & Final PDWS (EPA, 1993a) \\
\hline Antimony, dissolved & $\mu \mathrm{g} / \mathrm{L}$ & 3 & 6 & Final PDWS (EPA, 1993a) \\
\hline Antimony, total recoverable & $\mu g / L$ & 3 & 6 & Final PDWS (EPA, 1993a) \\
\hline Antimony-125 & $\mathrm{pCi} / \mathrm{L}$ & $1.5 \mathrm{E}+02$ & $3 E+02$ & Interim Final PDWS (EPA, 1977) \\
\hline Aramite & $\mu g / L$ & 50 & 100 & EPA Method 8270 \\
\hline Arsenic & $\mu \mathrm{g} / \mathrm{L}$ & 25 & 50 & Final PDWS (EPA, 1993a) \\
\hline Arsenic, dissolved & $\mu \mathrm{g} / \mathrm{L}$ & 25 & 50 & Final PDWS (EPA, 1993a) \\
\hline Arsenic, total recoverable & $\mu g / L$ & 25 & 50 & Final PDWS (EPA, 1993a) \\
\hline Asbestos & Fibers/L & $3,500,000$ & $7,000,000$ & Final PDWS (EPA, 1993a) \\
\hline Atrazine & $\mu g / L$ & 1.5 & 3 & Final PDWS (EPA, 1993a) \\
\hline Azobenzene & $\mu g / L$ & 50 & 100 & EPA Method 625 \\
\hline Barium & $\mu \mathrm{g} / \mathrm{L}$ & 1,000 & 2,000 & Final PDWS (EPA, 1993a) \\
\hline Barium, dissolved & $\mu g / L$ & 1,000 & 2,000 & Final PDWS (EPA, 1993a) \\
\hline Barium, total recoverable & $\mu g / L$ & 1,000 & 2,000 & Final PDWS (EPA, 1993a) \\
\hline Barium-140 & $\mathrm{pCi} / \mathrm{L}$ & $4.5 E+01$ & $9 \mathrm{E}+01$ & Interim Final PDWS (EPA, 1977) \\
\hline Benzene & $\mu \mathrm{g} / \mathrm{L}$ & 2.5 & 5 & Final PDWS (EPA, 1993a) \\
\hline alpha-Benzene hexachloride & $\mu g / L$ & 0.25 & 0.5 & EPA Method 8080 \\
\hline beta-Benzene hexachloride & $\mu g / L$ & 0.25 & 0.5 & EPA Method 8080 \\
\hline delta-Benzene hexachloride & $\mu \mathrm{g} / \mathrm{L}$ & 0.25 & 0.5 & EPA Method 8080 \\
\hline Benzidine & $\mu g / L$ & 250 & 500 & EPA Method 8270 \\
\hline Benzo[a]anthracene & $\mu g / L$ & 0.05 & 0.1 & Proposed PDWS (EPA, 1990) \\
\hline Benzo[b]fluoranthene & $\mu g / L$ & 0.1 & 0.2 & Proposed PDWS (EPA, 1990) \\
\hline Benzo[k]fluoranthene & $\mu g / L$ & 0.1 & 0.2 & Proposed PDWS (EPA, 1990) \\
\hline Benzoic acid & $\mu \mathrm{g} / \mathrm{L}$ & 250 & 500 & EPA Method $8270^{\circ}$ \\
\hline Benzo[g,h,i]perylene & $\mu \mathrm{g} / \mathrm{L}$ & 50 & 100 & EPA Method 8270 \\
\hline Benzo[a]pyrene & $\mu \mathrm{g} / \mathrm{L}$ & 0.1 & 0.2 & Final PDWS (EPA, 1993a) \\
\hline 1,4-Benzoquinone & $\mu g / L$ & 50 & 100 & EPA Method 8270 \\
\hline Benzyl alcohol & $\mu g / L$ & 50 & 100 & EPA Method 8270 \\
\hline Beryllium & $\mu g / L$ & 2 & 4 & Final PDWS (EPA, 1993a) \\
\hline Beryllium, dissolved & $\mu g / L$ & 2 & 4 & Final PDWS (EPA. 1993a) \\
\hline Beryllium, total recoverable & $\mu \mathrm{g} / \mathrm{L}$ & 2 & 4 & Final PDWS (EPA, 1993a) \\
\hline Beryllium-7 & $\mathrm{pCi} / \mathrm{L}$ & $3 E+03$ & $6 E+03$ & Interim Final PDWS (EPA, 1977) \\
\hline Bis(2-chloroethoxy) methane & $\mu g / L$ & 50 & 100 & EPA Method 8270 \\
\hline Bis(2-chloroethyl) ether & $\mu g / L$ & 50 & 100 & EPA Method 8270 \\
\hline Bis(2-chloroisopropyl) ether & $\mu g / L$ & 50 & 100 & EPA Method 8270 \\
\hline Bis(chloromethyl) ether & $\mu g / L$ & 50 & 100 & EPA Method 8270 \\
\hline Bis(2-ethylhexyl) phthalate & $\mu g / L$ & 3 & 6 & Final PDWS (EPA, 1993a) \\
\hline Bismuth-214 & $\mathrm{pCi} / \mathrm{L}$ & $9.4 E+03$ & $1.89 E+04$ & Proposed PDWS (EPA, 1991) \\
\hline Boron & $\mu g / L$ & 150 & 300 & EPA Method 6010 \\
\hline Boron, dissolved & $\mu g / L$ & 150 & 300 & EPA Method 6010 \\
\hline Boron, total recoverable & $\mu g / L$ & 150 & 300 & EPA Method 6010 \\
\hline Bromide & $\mu g / L$ & 5,000 & 10,000 & EPA Method 300.0 \\
\hline Bromodichloromethane & $\mu g / L$ & 50 & 100 & Final PDWS (EPA, 1993a) \\
\hline Bromoform & $\mu g / L$ & 50 & 100 & Final PDWS (EPA, 1993a) \\
\hline Bromomethane (Methyl bromide) & $\mu g / L$ & 5 & 10 & EPA Method 8240 \\
\hline 4-Bromophenyl phenyl ether & $\mu g / L$ & 50 & 100 & EPA Method 8270 \\
\hline Butylbenzyl phthalate & & No & No flag & Set by EPD/EMS \\
\hline 2-sec-Butyl-4,6-dinitrophenol & $\mu g / L$ & 3.5 & 7 & Final PDWS (EPA, 1993a) \\
\hline
\end{tabular}




Analyte
Cadmium
Cadmium, dissolved
Cadmium, total recoverable
Calcium
Calcium, dissolved
Calcium, total recoverable
Carbofuran
Carbon-14
Carbonate
Carbon disulfide
Carbon tetrachloride
Cerium-141
Cerium-144
Cesium-134
Cesium-137
Chlordane
Chloride
4-Chloroaniline
Chlorobenzene
Chlorobenzilate
4-Chloro-m-cresol
Chloroethane
Chloroethene (Vinyl chloride)
Chloroethyl vinyl ether
2-Chloroethyl vinyl ether
Chloroform
Chloromethane (Methyl chloride)
2-Chloronaphthalene
2-Chlorophenol
4-Chlorophenyl phenyl ether
Chloroprene
Chromium
Chromium, dissolved
Chromium, total recoverable
Chromium-51
Chrysene
Cobalt
Cobalt, dissolved
Cobalt, total recoverable
Cobalt-57
Cobalt-58
Cobalt-60
Color
Copper
Copper, dissolved
Copper, total recoverable
Corrosivity
m-Cresol (3-Methylphenol)
o-Cresol (2-Methylphenol)
p-Cresol (4-Methylphenol)
Curium-242
Curium-243
Curium-243/244e
Curium-244
Curium-245/246e

\begin{tabular}{|c|c|c|c|}
\hline Unit & Flag 1 & Flag 2 & Source $^{\mathrm{a}}$ \\
\hline$\mu g / L$ & 2.5 & 5 & Final PDWS (EPA, 1993a) \\
\hline$\mu \mathrm{g} / \mathrm{L}$ & 2.5 & 5 & Final PDWS (EPA, 1993a) \\
\hline$\mu g / L$ & 2.5 & 5 & Final PDWS (EPA, 1993a) \\
\hline & No flag & No flag & Set by EPD/EMS \\
\hline & No flag & No flag & Set by EPD/EMS \\
\hline & No flag & No flag & Set by EPD/EMS \\
\hline$\mu \mathrm{g} / \mathrm{L}$ & & & Final PDWS (EPA, 1993a) \\
\hline pCi/L & $\begin{array}{l}1 E+03 \\
\text { No flag }\end{array}$ & $2 E+03$ & Interim Final PDWS (EPA, 1977) \\
\hline$\mu \mathrm{g} / \mathrm{L}$ & $\begin{array}{l}\text { No flag } \\
5\end{array}$ & $\begin{array}{l}\text { No flag } \\
10\end{array}$ & $\begin{array}{l}\text { Set by EPD/EMS } \\
\text { EPA Method } 8240\end{array}$ \\
\hline$\mu \mathrm{g} / \mathrm{L}$ & 2.5 & 5 & Final PDWS (EPA, 1993a) \\
\hline $\mathrm{pCi} / \mathrm{L}$ & $1.5 \mathrm{E}+02$ & $3 E+02$ & Interim Final PDWS (EPA, 1977) \\
\hline $\mathrm{pCi} / \mathrm{L}$ & $1.31 E+02$ & 2.61E+02 & Proposed PDWS (EPA, 1991) \\
\hline $\mathrm{pCi/L}$ & 4.07E+01 & 8.13E+01 & Proposed PDWS (EPA, 1991) \\
\hline $\mathrm{pCi} / \mathrm{L}$ & $1 E+02$ & $2 E+02$ & Interim Final PDWS (EPA, 1977) \\
\hline$\mu \mathrm{g} / \mathrm{L}$ & 1 & 2 & Final PDWS (EPA, 1993a) \\
\hline$\mu \mathrm{g} / \mathrm{L}$ & 125,000 & 250,000 & SDWS (EPA, 1993b) \\
\hline$\mu \mathrm{g} / \mathrm{L}$ & 50 & 100 & EPA Method 8270 \\
\hline$\mu \mathrm{g} / \mathrm{L}$ & 50 & 100 & Final PDWS (EPA, 1993a) \\
\hline$\mu \mathrm{g} / \mathrm{L}$ & 50 & 100 & EPA Method 8270 \\
\hline$\mu \mathrm{g} / \mathrm{L}$ & 50 & 100 & EPA Method 8270 \\
\hline$\mu \mathrm{g} / \mathrm{L}$ & 5 & 10 & EPA Method 8240 \\
\hline$\mu \mathrm{g} / \mathrm{L}$ & 1 & 2 & Final PDWS (EPA, 1993a) \\
\hline$\mu \mathrm{g} / \mathrm{L}$ & 5 & 10 & EPA Method 8240 \\
\hline$\mu g / L$ & 5 & 10 & EPA Method 8240 \\
\hline$\mu g / L$ & 50 & 100 & Final PDWS (EPA, 1993a) \\
\hline$\mu g / L$ & 5 & 10 & EPA Method 8240 \\
\hline$\mu \mathrm{g} / \mathrm{L}$ & 50 & 100 & EPA Method 8240 \\
\hline$\mu \mathrm{g} / \mathrm{L}$ & 50 & 100 & EPA Method 8270 \\
\hline$\mu g / L$ & 50 & 100 & EPA Method 8270 \\
\hline$\mu g / L$ & 1,000 & 2,000 & EPA Method 8240 \\
\hline$\mu g / L$ & 50 & 100 & Final PDWS (EPA, 1993a) \\
\hline$\mu \mathrm{g} / \mathrm{L}$ & 50 & 100 & Final PDWS (EPA, 1993a) \\
\hline$\mu \mathrm{g} / \mathrm{L}$ & 50 & 100 & Final PDWS (EPA, 1993a) \\
\hline $\mathrm{pCi} / \mathrm{L}$ & $3 E+03$ & $6 E \div 03$ & Interim Final PDWS (EPA, 1977) \\
\hline$\mu g / L$ & 0.1 & 0.2 & Proposed PDWS (EPA, 1990) \\
\hline$\mu g / L$ & 20 & 40 & EPA Method 6010 \\
\hline$\mu \mathrm{g} / \mathrm{L}$ & 20 & 40 & EPA Method 6010 \\
\hline$\mu \mathrm{g} / \mathrm{L}$ & 20 & 40 & EPA Method 6010 \\
\hline $\mathrm{pCi} / \mathrm{L}$ & $5 E+02$ & $1 E+03$ & Interim Final PDWS (EPA, 1977) \\
\hline pCilL & $4.5 E+03$ & $9 E+03$ & Interim Final PDWS (EPA, 1977) \\
\hline pCilL & $\begin{array}{l}5 E+01 \\
\text { No flag }\end{array}$ & $\begin{array}{l}1 E+02 \\
\text { No flag }\end{array}$ & $\begin{array}{l}\text { Interim Final PDWS (EPA, 1977) } \\
\text { Set by EPD/EMS }\end{array}$ \\
\hline$\mu \mathrm{g} / \mathrm{L}$ & 500 & 1,000 & Final PDWS (SCDHEC, 1981) \\
\hline$\mu \mathrm{g} / \mathrm{L}$ & 500 & 1,000 & Final PDWS (SCDHEC, 1981) \\
\hline$\mu \mathrm{g} / \mathrm{L}$ & 500 & 1,000 & Final PDWS (SCDHEC, 1981) \\
\hline & No flag & No flag & Set by EPD/EMS \\
\hline$\mu g /$ & 50 & 100 & EPA Method 8270 \\
\hline$\mu \mathrm{g} / \mathrm{L}$ & 50 & 100 & EPA Method 8270 \\
\hline$\mu \mathrm{g} / \mathrm{L}$ & 50 & 100 & EPA Method 8270 \\
\hline $\mathrm{pCi} / \mathrm{L}$ & $6.65 E+01$ & $1.33 E+02$ & Proposed PDWS (EPA, 1991) \\
\hline $\mathrm{pCi} / \mathrm{L}$ & 4.15E+00 & $8.3 E+00$ & Proposed PDWS (EPA, 1991) \\
\hline $\mathrm{pCi} / \mathrm{L}$ & 4.15E+00 & $8.3 E+00$ & Proposed PDWS (EPA, 1991) \\
\hline $\mathrm{pCi} / \mathrm{L}$ & $4.92 E+00$ & $9.84 \mathrm{E}+00$ & Proposed PDWS (EPA, 1991) \\
\hline $\mathrm{pCi} / \mathrm{L}$ & $3.12 E+00$ & $6.23 E+00$ & Proposed PDWS (EPA, 1991) \\
\hline
\end{tabular}




\begin{tabular}{|c|c|c|c|c|}
\hline Analyte & $\underline{\text { Unit }}$ & Flag 1 & Flag 2 & Source $^{a}$ \\
\hline Curium-246 & $\mathrm{pCi} / \mathrm{L}$ & $3.14 E+00$ & $6.27 E+00$ & Proposed PDWS (EPA, 1991) \\
\hline Cyanide & $\mu \mathrm{g} / \mathrm{L}$ & 100 & 200 & Final PDWS (EPA, 1993a) \\
\hline Dalapon ${ }^{\mathbf{b}}$ & $\mu g / L$ & 100 & 200 & Final PDWS (EPA, 1993a) \\
\hline$p, p^{\prime}-D D D$ & $\mu g / L$ & 0.5 & 1 & EPA Method 8080 \\
\hline$p, p^{\prime}-D D E$ & $\mu g / L$ & 0.5 & 1 & EPA Method 8080 \\
\hline p,p'-DDT & $\mu \mathrm{g} / \mathrm{L}$ & 0.5 & 1 & EPA Method 8080 \\
\hline Diallate & $\mu g / L$ & 50 & 100 & EPA Method 8270 \\
\hline Dibenz[a, $h]$ anthracene & $\mu \mathrm{g} / \mathrm{L}$ & 0.15 & 0.3 & Proposed PDWS (EPA, 1990) \\
\hline Dibenzofuran & $\mu g / L$ & 50 & 100 & EPA Method 8270 \\
\hline Dibromochloromethane & $\mu \mathrm{g} / \mathrm{L}$ & 50 & 100 & Final PDWS (EPA, 1993a) \\
\hline 1,2-Dibromo-3-chloropropane & $\mu g / L$ & 0.1 & 0.2 & Final PDWS (EPA, 1993a) \\
\hline 1,2-Dibromoethane & $\mu \mathrm{g} / \mathrm{L}$ & 0.025 & 0.05 & Final PDWS (EPA, 1993a) \\
\hline $\begin{array}{l}\text { Dibromomethane } \\
\text { (Methylene bromide) }\end{array}$ & $\mu g / L$ & 5 & 10 & EPA Method 8240 \\
\hline Di-n-butyl phthalate & & No flag & No flag & Set by EPD/EMS \\
\hline 1,2-Dichlorobenzene & $\mu \mathrm{g} / \mathrm{L}$ & 300 & 600 & Final PDWS (EPA, 1993a) \\
\hline 1,3-Dichlorobenzene & $\mu g / L$ & 50 & 100 & EPA Method 8270 \\
\hline 1,4-Dichlorobenzene & $\mu \mathrm{rg} / \mathrm{L}$ & 37.5 & 75 & Final PDWS (EPA, 1993a) \\
\hline 3,3'-Dichlorobenzidine & $\mu g / L$ & 50 & 100 & EPA Method 8270 \\
\hline trans-1,4-Dichloro-2-butene & $\mu g / L$ & 150 & 300 & EPA Method 8240 \\
\hline Dichlorodiffuoromethane & $\mu g / L$ & 5 & 10 & EPA Method 8240 \\
\hline 1,1-Dichloroethane & $\mu g / L$ & 5 & 10 & EPA Method 8240 \\
\hline 1,2-Dichloroethane & $\mu \mathrm{g} / \mathrm{L}$ & 2.5 & 5 & Final PDWS (EPA, 1993a) \\
\hline 1,1-Dichloroethylene & $\mu g / L$ & 3.5 & 7 & Final PDWS (EPA, 1993a) \\
\hline 1,2-Dichloroethylene & $\mu \mathrm{g} / \mathrm{L}$ & 25 & 50 & Final PDWS (EPA, 1993a) \\
\hline cis-1,2-Dichloroethylene & $\mu g / L$ & 35 & 70 & Final PDWS (EPA, 1993a) \\
\hline trans-1,2-Dichloroethylene & $\mu g / L$ & 50 & 100 & Final PDWS (EPA, 1993a) \\
\hline $\begin{array}{l}\text { Dichloromethane } \\
\text { (Methylene chloride) }\end{array}$ & $\mu \mathrm{g} / \mathrm{L}$ & 2.5 & 5 & Final PDWS (EPA, 1993a) \\
\hline 2,4-Dichlorophenol & $\mu \mathrm{g} / \mathrm{L}$ & 50 & 100 & EPA Method 8270 \\
\hline 2,6-Dichlorophenol & $\mu \mathrm{g} / \mathrm{L}$ & 50 & 100 & EPA Method 8270 \\
\hline 2,4-Dichlorophenoxyacetic acid & $\mu \mathrm{g} / \mathrm{L}$ & 35 & 70 & Final PDWS (EPA, 1993a) \\
\hline 1,2-Dichloropropane & $\mu g / L$ & 2.5 & 5 & Final PDWS (EPA, 1993a) \\
\hline cis-1,3-Dichloropropene & $\mu \mathrm{g} / \mathrm{L}$ & 5 & 10 & EPA Method 8240 \\
\hline trans-1,3-Dichloropropene & $\mu g / L$ & 5 & 10 & EPA Method 8240 \\
\hline Dieldrin & $\mu \mathrm{g} / \mathrm{L}$ & 2.5 & 5 & EPA Method 8080 \\
\hline Di(2-ethylhexyl) adipate & $\mu \mathrm{g} / \mathrm{L}$ & 200 & 400 & Final PDWS (EPA, 1993a) \\
\hline Diethyl phthalate & & No flag & No flag & Set by EPD/EMS \\
\hline Dimethoate & $\mu \mathrm{g} / \mathrm{L}$ & 50 & 100 & EPA Method 8270 \\
\hline p-Dimethylaminoazobenzene & $\mu \mathrm{g} / \mathrm{L}$ & 50 & 100 & EPA Method 8270 \\
\hline p-(Dimethylamino)ethylbenzene & $\mu \mathrm{g} / \mathrm{L}$ & 50 & 100 & EPA Method 8270 \\
\hline 7,12-Dimethylbenz[a]anthracene & $\mu \mathrm{g} / \mathrm{L}$ & 50 & 100 & EPA Method 8270 \\
\hline 3,3'-Dimethylbenzidine & $\mu \mathrm{g} / \mathrm{L}$ & 50 & 100 & EPA Method 8270 \\
\hline a,a-Dimethylphenethylamine & $\mu \mathrm{g} / \mathrm{L}$ & 50 & 100 & EPA Method 8270 \\
\hline 2,4-Dimethyl phenol & $\mu \mathrm{g} / \mathrm{L}$ & 50 & 100 & EPA Method 8270 \\
\hline Dimethyl phthalate & & No flag & No flag & Set by EPD/EMS \\
\hline 1,3-Dinitrobenzene & $\mu \mathrm{g} / \mathrm{L}$ & & 100 & EPA Method 8270 \\
\hline 2,4-Dinitrophenol & $\mu \mathrm{g} / \mathrm{L}$ & 250 & 500 & EPA Method 8270 \\
\hline 2,4-Dinitrotoluene & $\mu g / L$ & 50 & 100 & EPA Method 8270 \\
\hline 2,6-Dinitrotoluene & $\mu \mathrm{g} / \mathrm{L}$ & 50 & 100 & EPA Method 8270 \\
\hline Di-n-octyl phthalate & & No & No flag & Set by EPD/EMS \\
\hline 1,4-Dioxane & $\mu g / L$ & 50 & 100 & EPA Method 8270 \\
\hline Diphenylamine & $\mu \mathrm{g} / \mathrm{L}$ & 50 & 100 & EPA Method 8270 \\
\hline 1,2-Diphenylhydrazine & $\mu g / L$ & 50 & 100 & EPA Method 8270 \\
\hline Diquat dibromide ${ }^{b}$ & $\mu \mathrm{g} / \mathrm{L}$ & 10 & 20 & Final PDWS (EPA, 1993a) \\
\hline
\end{tabular}




\begin{tabular}{|c|c|c|c|c|}
\hline Analyte & $\underline{\text { Unit }}$ & Flag 1 & Flag 2 & $\underline{\text { Source }}^{\mathrm{a}}$ \\
\hline Dissolved organic carbon & $\mu \mathrm{g} / \mathrm{L}$ & 5,000 & 10,000 & EPA Method 9060 \\
\hline Disulfoton & $\mu \mathrm{g} / \mathrm{L}$ & & & EPA Method 8270 \\
\hline $\begin{array}{l}\text { En } \\
\text { Endosulfan I }\end{array}$ & $\mu \mathrm{g} / \mathrm{L}$ & $\begin{array}{l}\text { No flag } \\
0.5\end{array}$ & $\begin{array}{l}\text { No flag } \\
1\end{array}$ & $\begin{array}{l}\text { Set by EPD/EMS } \\
\text { EPA Method } 8080\end{array}$ \\
\hline Endosulfan II & $\mu \mathrm{g} / \mathrm{L}$ & 0.5 & 1 & EPA Method 8080 \\
\hline Endosulfan sulfate & $\mu \mathrm{g} / \mathrm{L}$ & 0.5 & 1 & EPA Method 8080 \\
\hline Endothali $^{\mathrm{b}}$ & $\mu g / L$ & 50 & 100 & Final PDWS (EPA, 1993a) \\
\hline Endrin & $\mu \mathrm{g} / \mathrm{L}$ & 1 & 2 & Final PDWS (EPA, 1993a) \\
\hline Endrin aldehyde & $\mu \mathrm{g} / \mathrm{L}$ & 0.5 & 1 & EPA Method 8080 \\
\hline Endrin ketone & & No flag & No flag & Set by EPD/EMS \\
\hline Ethylbenzene & $\mu \mathrm{g} / \mathrm{L}$ & 350 & 700 & Final PDWS (EPA, 1993a) \\
\hline Ethyl methacrylate & $\mu g / L$ & 50 & 100 & EPA Method 8270 \\
\hline Ethyl methanesulfonate & $\mu \mathrm{g} / \mathrm{L}$ & 50 & 100 & EPA Method 8270 \\
\hline Europium-152 & $\mathrm{pCi} / \mathrm{L}$ & $3 E+01$ & $6 E+01$ & Interim Final PDWS (EPA, 1977) \\
\hline Europium-154 & $\mathrm{pCi} / \mathrm{L}$ & $1 E+02$ & $2 E+02$ & Interim Final PDWS (EPA, 1977) \\
\hline Europium-155 & $\mathrm{pCi} / \mathrm{L}$ & $3 E+02$ & $6 E+02$ & Interim Final PDWS (EPA, 1977) \\
\hline Famphur & $\mu \mathrm{g} / \mathrm{L}$ & 50 & 100 & EPA Method 8270 \\
\hline Fluoranthene & $\mu \mathrm{g} / \mathrm{L}$ & 50 & 100 & EPA Method 8270 \\
\hline Fluorene & $\mu \mathrm{g} / \mathrm{L}$ & 50 & 100 & EPA Method 8270 \\
\hline Fluoride & $\mu \mathrm{g} / \mathrm{L}$ & 2,000 & 4,000 & Final PDWS (EPA, 1993a) \\
\hline Glyphosate $^{b}$ & $\mu \mathrm{g} / \mathrm{L}$ & 350 & 700 & Final PDWS (EPA, 1993a) \\
\hline Gross alpha & pCill & $7.5 E+00$ & $1.5 \mathrm{E}+01$ & Final PDWS (EPA, 1993a) \\
\hline Heptachior & $\mu \mathrm{g} / \mathrm{L}$ & 0.2 & 0.4 & Final PDWS (EPA, 1993a) \\
\hline Heptachlor epoxide & $\mu g / L$ & 0.1 & 0.2 & Final PDWS (EPA, 1993a) \\
\hline $\begin{array}{l}\text { Heptachlorodibenzo-p-dioxin } \\
\text { isomers }\end{array}$ & $\mu \mathrm{g} / \mathrm{L}$ & 0.00325 & 0.0065 & EPA Method 8280 \\
\hline 1,2,3,4,6,7,8-HPCDD & $\mu \mathrm{g} / \mathrm{L}$ & 0.00325 & 0.0065 & EPA Method 8280 \\
\hline $\begin{array}{l}\text { Heptachlorodibenzo-p-furan } \\
\text { isomers }\end{array}$ & $\mu g / L$ & 0.00225 & 0.0045 & EPA Method $\mathbf{8 2 8 0}$ \\
\hline $1,2,3,4,6,7,8-\mathrm{HPCDF}$ & $\mu \mathrm{g} / \mathrm{L}$ & 0.00225 & 0.0045 & EPA Method 8280 \\
\hline Hexachlorobenzene & $\mu \mathrm{g} / \mathrm{L}$ & 0.5 & & Final PDWS (EPA, 1993a) \\
\hline Hexachlorobutadiene & $\mu \mathrm{g} / \mathrm{L}$ & 50 & 100 & EPA Method 8270 \\
\hline Hexachlorocyclopentadiene & $\mu \mathrm{g} / \mathrm{L}$ & 25 & 50 & Final PDWS (EPA, 1993a) \\
\hline $\begin{array}{l}\text { Hexachlorodibenzo-p-dioxin } \\
\text { isomers }\end{array}$ & $\mu g / L$ & 0.00225 & 0.0045 & EPA Method 8280 \\
\hline $1,2,3,4,7,8-H \times C D D$ & $\mu \mathrm{g} / \mathrm{L}$ & 0.00225 & 0.0045 & EPA Method 8280 \\
\hline $\begin{array}{l}\text { Hexachlorodibenzo-p-furan } \\
\text { isomers }\end{array}$ & $\mu \mathrm{g} / \mathrm{L}$ & 0.002 & 0.004 & EPA Method 8280 \\
\hline $1,2,3,4,7,8-H X C D F$ & $\mu \mathrm{g} / \mathrm{L}$ & 0.002 & 0.004 & EPA Method 8280 \\
\hline Hexachloroethane & $\mu \mathrm{g} / \mathrm{L}$ & 50 & 100 & EPA Method 8270 \\
\hline Hexachlorophene & $\mu g / L$ & 250 & 500 & EPA Method 8270 \\
\hline Hexachloropropene & $\mu \mathrm{g} / \mathrm{L}$ & 50 & 100 & EPA Method 8270 \\
\hline 2-Hexanone & $\mu \mathrm{g} / \mathrm{L}$ & 50 & 100 & EPA Method 8240 \\
\hline Indeno[1,2,3-c,d]pyrene & $\mu \mathrm{g} / \mathrm{L}$ & 50 & 100 & EPA Method 8270 \\
\hline lodine & $\mu \mathrm{g} / \mathrm{L}$ & 250 & 500 & APHA Method 415A \\
\hline lodine-129 & $\mathrm{pCi} / \mathrm{L}$ & $5 E-01$ & $1 E+00$ & Interim Final PDWS (EPA, 1977) \\
\hline lodine-131 ${ }^{c}$ & $\mathrm{pCi} / \mathrm{L}$ & $1.5 E+00$ & $3 E+00$ & Interim Final PDWS (EPA, 1977) \\
\hline lodomethane (Methyl iodide) & $\mu \mathrm{g} / \mathrm{L}$ & 75 & 150 & EPA Method 8240 \\
\hline Iron & $\mu \mathrm{g} / \mathrm{L}$ & 150 & 300 & SDWS (EPA, 1993b) \\
\hline Iron, dissolved & $\mu g / L$ & 150 & 300 & SDWS (EPA, 1993b) \\
\hline Iron, total recoverable & $\mu g / L$ & 150 & 300 & SDWS (EPA, 1993b) \\
\hline Iron-55c & $\mathrm{pCi/L}$ & $1 E+03$ & $2 E+03$ & Interim Final PDWS (EPA, 1977) \\
\hline Iron- $59^{c}$ & pCi/L & $1 \mathrm{E}+02$ & $2 E+02$ & Interim Final PDWS (EPA, 1977) \\
\hline Isobutyl alcohol & $\mu \mathrm{g} / \mathrm{L}$ & 500 & 1,000 & EPA Method 8240 \\
\hline Isodrin & $\mu g / L$ & 50 & 100 & EPA Method 8270 \\
\hline
\end{tabular}




\begin{tabular}{|c|c|c|c|c|}
\hline Analyte & $\underline{\text { Unit }}$ & Flag 1 & Flag 2 & Source $^{a}$ \\
\hline Isophorone & $\mu g / L$ & 50 & 100 & EPA Method 8270 \\
\hline Isosafrole & $\mu \mathrm{g} / \mathrm{L}$ & 50 & 100 & EPA Method 8270 \\
\hline Kepone & $\mu g / L$ & 50 & 100 & EPA Method 8270 \\
\hline Lanthanum-140 $\mathrm{C}$ & pCill & $3 E+01$ & $6 E+01$ & Interim Final PDWS (EPA, 1977) \\
\hline Lead & $\mu g / L$ & 25 & 50 & Final PDWS (SCDHEC, 1981) \\
\hline Lead, dissolved & $\mu g / L$ & 25 & 50 & Final PDWS (SCDHEC, 1981) \\
\hline Lead, total recoverable & $\mu g / L$ & 25 & 50 & Final PDWS (SCDHEC, 1981) \\
\hline Lead-212 & pCill & $6.2 E+01$ & $1.23 E+02$ & Proposed PDWS (EPA, 1991) \\
\hline Lindane & $\mu \mathrm{g} / \mathrm{L}$ & 0.1 & 0.2 & Final PDWS (EPA, 1993a) \\
\hline Lithium & $\mu \mathrm{g} / \mathrm{L}$ & 25 & 50 & EPA Method 6010 \\
\hline Lithium, dissolved & $\mu \mathrm{g} / \mathrm{L}$ & 25 & 50 & EPA Method 6010 \\
\hline Lithium, total recoverable & $\mu \mathrm{gg} / \mathrm{L}$ & 25 & 50 & EPA Method 6010 \\
\hline Magnesium & & No flag & No flag & Set by EPD/EMS \\
\hline Magnesium, dissolved & & No flag & No flag & Set by EPD/EMS \\
\hline Magnesium, total recoverable & & No flag & No flag' & Set by EPD/EMS \\
\hline Manganese & $\mu g / L$ & 25 & 50 & SDWS (EPA, 1993b) \\
\hline Manganese, dissolved & $\mu \mathrm{g} / \mathrm{L}$ & 25 & 50 & SDWS (EPA, 1993b) \\
\hline Manganese, total recoverable & $\mu g / L$ & 25 & 50 & SDWS (EPA, 1993b) \\
\hline Manganese-54 & pCi/L & $1.5 \mathrm{E}+02$ & $3 E+02$ & Interim Final PDWS (EPA, 1977) \\
\hline Mercury & $\mu g / L$ & 1 & 2 & Final PDWS (EPA, 1993a) \\
\hline Mercury, dissolved & $\mu g / L$ & 1 & 2 & Final PDWS (EPA, 1993a) \\
\hline Mercury, total recoverable & $\mu g / L$ & 1 & 2 & Final PDWS (EPA, 1993a) \\
\hline Methacrylonitrile & $\mu g / L$ & 250 & 500 & EPA Method 8240 \\
\hline Methapyrilene & $\mu g / L$ & 50 & 100 & EPA Method 8270 \\
\hline Methoxychlor & $\mu g / L$ & 20 & 40 & Final PDWS (EPA, 1993a) \\
\hline 3-Methylcholanthrene & $\mu g / L$ & 50 & 100 & EPA Method 8270 \\
\hline 2-Methyl-4,6-dinitrophenol & $\mu \mathrm{g} / \mathrm{L}$ & 250 & 500 & EPA Method 8270 \\
\hline Methyl ethyl ketone & & No flag & No flag & Set by EPD/EMS \\
\hline Methyl isobutyl ketone & & No flag & No flag & Set by EPD/EMS \\
\hline Methyl methacrylate & $\mu g / L$ & 50 & 100 & EPA Method 8270 \\
\hline Methyl methanesulfonate & $\mu g / L$ & 50 & 100 & EPA Method 8270 \\
\hline 2-Methylnaphthalene & $\mu g / L$ & 50 & 100 & EPA Method 8270 \\
\hline Molybdenum & $\mu g / L$ & 250 & 500 & EPA Method 6010 \\
\hline Molybdenum, dissolved & $\mu g / L$ & 250 & 500 & EPA Method 6010 \\
\hline Molybdenum, total recoverable & $\mu g / L$ & 250 & 500 & EPA Method 6010 \\
\hline Naphthalene & $\mu g / L$ & 50 & 100 & EPA Method 8270 \\
\hline 1,4-Naphthoquinone & $\mu g / L$ & 50 & 100 & EPA Method 8270 \\
\hline 1-Naphthylamine & $\mu g / L$. & 50 & 100 & EPA Method 8270 \\
\hline 2-Naphthylamine & $\mu \mathrm{g} / \mathrm{L}$ & 50 & 100 & EPA Method 8270 \\
\hline Neptunium-237 & $\mathrm{pCi} / \mathrm{L}$ & $3.53 E+00$ & 7.06E+00 & Proposed PDWS (EPA, 1991) \\
\hline Nickel & $\mu g / L$ & 50 & 100 & Final PDWS (EPA, 1993a) \\
\hline Nickel, dissolved & $\mu g / L$ & 50 & 100 & Final PDWS (EPA, 1993a) \\
\hline Nickel, total recoverable & $\mu g / L$ & 50 & 100 & Final PDWS (EPA, 1993a) \\
\hline Nickel-59c & pCi/L & $1.5 E+02$ & $3 E+02$ & Interim Final PDWS (EPA, 1977) \\
\hline Nickel-63 ${ }^{c}$ & $\mathrm{pCi} / \mathrm{L}$ & $2.5 E+01$ & $5 E+01$ & Interim Final PDWS (EPA, 1977) \\
\hline Niobium-95 & $\mathrm{pCi} / \mathrm{L}$ & $1.5 E+02$ & 3.E+02: & Interim Final PDWS (EPA, 1977) \\
\hline Nitrate as nitrogen & $\mu g / L$ & 5,000 & 10,000 & Final PDWS (EPA, 1993a) \\
\hline Nitrate-nitrite as nitrogen & $\mu g / L$ & 5,000 & 10,000 & Final PDWS (EPA, 1993a) \\
\hline Nitrite as nitrogen & $\mu g / L$ & 500 & 1,000 & Final PDWS (EPA, 1993a) \\
\hline m-Nitroaniline & $\mu g / L$ & 50 & 100 & EPA Method 8270 \\
\hline o-Nitroaniline & $\mu \mathrm{g} / \mathrm{L}$ & 50 & 100 & EPA Method 8270 \\
\hline p-Nitroaniline & $\mu g / L$ & 50 & 100 & EPA Method 8270 \\
\hline Nitrobenzene & $\mu g / L$ & 50 & 100 & EPA Method 8270 \\
\hline Nitrogen by Kjeldahl method & $\mu g / L$ & 500 & 1,000 & EPA Method 351.2 \\
\hline 2-Nitrophenol & $\mu g / L$ & 50 & 100 & EPA Method 8270 \\
\hline
\end{tabular}




\begin{tabular}{|c|c|c|c|c|}
\hline Analyte & Unit & Flag 1 & Flag 2 & Source $^{a}$ \\
\hline 4-Nitrophenol & $\mu \mathrm{g} / \mathrm{L}$ & 50 & 100 & EPA Method 8270 \\
\hline 4-Nitroquinoline-1-oxide & $\mu \mathrm{g} / \mathrm{L}$ & 50 & 100 & EPA Method 8270 \\
\hline N-Nitrosodi-n-butylamine & $\mu \mathrm{g} / \mathrm{L}$ & 50 & 100 & EPA Method 8270 \\
\hline N-Nitrosodiethylamine & $\mu \mathrm{g} / \mathrm{L}$ & 50 & 100 & EPA Method 8270 \\
\hline N-Nitrosodimethylamine & $\mu g / L$ & 50 & 100 & EPA Method 8270 \\
\hline N-Nitrosodiphenylamine & $\mu \mathrm{g} / \mathrm{L}$ & 50 & 100 & EPA Method 8270 \\
\hline N-Nitrosodipropylamine & $\mu \mathrm{g} / \mathrm{L}$ & 50 & 100 & EPA Method 8270 \\
\hline N-Nitrosomethylethylamine & $\mu \mathrm{g} / \mathrm{L}$ & 50 & 100 & EPA Method 8270 \\
\hline N-Nitrosomorpholine & $\mu \mathrm{g} / \mathrm{L}$ & 50 & 100 & EPA Method 8270 \\
\hline N-Nitrosopiperidine & $\mu \mathrm{g} / \mathrm{L}$ & 50 & 100 & EPA Method 8270 \\
\hline N-Nitrosopyrrolidine & $\mu g / L$ & 50 & 100 & EPA Method 8270 \\
\hline 5-Nitro-o-toluidine & $\mu g / L$ & 50 & 100 & EPA Method 8270 \\
\hline Nonvolatile beta & pCill & $2.5 E+01$ & $5 E+01$ & Interim Final PDWS (EPA, 1977) \\
\hline $\begin{array}{l}\text { Octachlorodibenzo-p-dioxin } \\
\text { isomers }\end{array}$ & $\mu g / L$ & 0.005 & 0.01 & EPA Method 8280 \\
\hline $\begin{array}{l}\text { Octachlorodibenzo-p-furan } \\
\text { isomers }\end{array}$ & $\mu g / L$ & 0.005 & 0.01 & EPA Method 8280 \\
\hline Odor & & No flag & No flag & Set by EPD/EMS \\
\hline Oil \& Grease & $\mu g / L$ & 5,000 & 10,000 & EPA Method 413.1 \\
\hline Oxamyl $\left.\right|^{b}$ & $\mu \mathrm{g} / \mathrm{L}$ & 100 & 200 & Final PDWS (EPA, 1993a) \\
\hline Parathion & $\mu \mathrm{g} / \mathrm{L}$ & 0.25 & 0.5 & EPA Method $8080^{\circ}$ \\
\hline Parathion methyl & $\mu \mathrm{g} / \mathrm{L}$ & 0.25 & 0.5 & EPA Method 8080 \\
\hline РСВ 1016 & $\mu g / L$ & 0.25 & 0.5 & Final PDWS (EPA, 1993a) \\
\hline PCB 1221 & $\mu \mathrm{g} / \mathrm{L}$ & 0.25 & 0.5 & Final PDWS (EPA, 1993a) \\
\hline PCB 1232 & $\mu g / L$ & 0.25 & 0.5 & Final PDWS (EPA, 1993a) \\
\hline PCB 1242 & $\mu \mathrm{g} / \mathrm{L}$ & 0.25 & 0.5 & Final PDWS (EPA, 1993a) \\
\hline PCB 1248 & $\mu \mathrm{g} / \mathrm{L}$ & 0.25 & 0.5 & Final PDWS (EPA, 1993a) \\
\hline PCB 1254 & $\mu g / L$ & 0.25 & 0.5 & Final PDWS (EPA, 1993a) \\
\hline PCB 1260 & $\mu g / L$ & 0.25 & 0.5 & Final PDWS (EPA, 1993a) \\
\hline PCB 1262 & $\mu \mathrm{g} / \mathrm{L}$ & 0.25 & 0.5 & Final PDWS (EPA, 1993a) \\
\hline Pentachlorobenzene & $\mu \mathrm{g} / \mathrm{L}$ & 50 & 100 & EPA Method 8270 \\
\hline $\begin{array}{l}\text { Pentachlorodibenzo-p-dioxin } \\
\text { isomers }\end{array}$ & $\mu \mathrm{g} / \mathrm{L}$ & 0.00275 & 0.0055 & EPA Method 8280 \\
\hline 1,2,3,7,8-PCDD & $\mu g / L$ & 0.00275 & 0.0055 & EPA Method 8280 \\
\hline $\begin{array}{l}\text { Pentachlorodibenzo-p-furan } \\
\text { isomers }\end{array}$ & $\mu g / L$ & 0.00275 & 0.0055 & EPA Method 8280 \\
\hline $1,2,3,7,8-P C D F$ & $\mu \mathrm{g} / \mathrm{L}$ & 0.00275 & 0.0055 & EPA Method 8280 \\
\hline Pentachloroethane & $\mu \mathrm{g} / \mathrm{L}$ & 50 & 100 & EPA Method 8270 \\
\hline Pentachloronitrobenzene & $\mu \mathrm{g} / \mathrm{L}$ & 50 & 100 & EPA Method 8270 \\
\hline Pentachlorophenol & $\mu g / L$ & 0.5 & 1 & Final PDWS (EPA, 1993a) \\
\hline $\mathrm{pH}$ & $\mathrm{pH}$ & 8 & 10 & Set by EPD/EMS \\
\hline $\mathrm{pH}$ & $\mathrm{pH}$ & 4 & 3 & Set by EPD/EMS \\
\hline Phenacetin & $\mu g / L$ & 50 & 100 & EPA Method 8270 \\
\hline Phenanthrene & $\mu \mathrm{g} / \mathrm{L}$ & 50 & 100 & EPA Method 8270 \\
\hline Phenol & $\mu \mathrm{g} / \mathrm{L}$ & 50 & 100 & EPA Method 8270 \\
\hline Phenols & $\mu \mathrm{g} / \mathrm{L}$ & 25 & 50 & EPA Method 420.1 \\
\hline p-Phenylenediamine & $\mu g / L$ & 50 & 100 & EPA Method 8270 \\
\hline Phorate & $\mu g / L$ & 0.5 & 1 & EPA Method 8080 \\
\hline Picloram ${ }^{b}$ & $\mu \mathrm{g} / \mathrm{L}$ & 250 & 500 & Final PDWS (EPA, 1993a) \\
\hline 2-Picoline & $\mu \mathrm{g} / \mathrm{L}$ & 50 & 100 & EPA Method 8270 \\
\hline Plutonium-238 & $\mathrm{pCi} / \mathrm{L}$ & $3.51 E+00$ & $7.02 E+00$ & Proposed PDWS (EPA, 1991) \\
\hline Plutonium-239 & $\mathrm{pCi} / \mathrm{L}$ & $3.11 E+01$ & $6.21 E+01$ & Proposed PDWS (EPA, 1991) \\
\hline Plutonium-239/240 ${ }^{\mathrm{e}}$ & $\mathrm{pCi} / \mathrm{L}$ & $3.11 E+01$ & $6.21 E+01$ & Proposed PDWS (EPA, 1991) \\
\hline Plutonium-240 & $\mathrm{pCi} / \mathrm{L}$ & $3.11 E+01$ & $6.22 E+01$ & Proposed PDWS (EPA, 1991) \\
\hline Plutonium-241 ${ }^{c}$ & $\mathrm{pCi} / \mathrm{L}$ & $3.13 E+01$ & $6.26 \mathrm{E}+01$ & Proposed PDWS (EPA 1991) \\
\hline
\end{tabular}


Analvie

Plutonium-242 ${ }^{c}$

Potassium

Potassium, dissolved

Potassium, total recoverable

Potassium-40

Promethium-144

Promethium-146

Promethium-147

Pronamid

Propionitrile

Pyrene

Pyridine

Radium (alpha-emitting) ${ }^{f}$

Radium-226

Radium-228

Radon-222

Ruthenium-103 ${ }^{c}$

Ruthenium-106

Safrole

Selenium

Selenium, dissolved

Selenium, total recoverable

Silica

Silica, dissolved

Silica, total recoverable

Silver

Silver, dissolved

Silver, total recoverable

Simazine ${ }^{b}$

Sodium

Sodium, dissolved

Sodium, total recoverable

Sodium-22

Specific conductance

Strontium-89

Strontium-89/90 ${ }^{\mathrm{e}}$

Strontium-90

Styrene

Sulfate

Sulfide

Sulfotepp

Surfactants

2,3,7,8-TCDD

2,3,7,8-TCDF

Technetium-99

1,2,4,5-Tetrachlorobenzene

Tetrachlorodibenzo-p-dioxin isomers

Tetrachlorodibenzo-p-furan isomers

$1,1,1,2-$ Tetrachloroethane

1,1,2,2-Tetrachloroethane

Tetrachloroethylene

2,3,4,6-Tetrachlorophenol

Thallium

\begin{tabular}{|c|c|c|}
\hline Unit & Flag 1 & Flag 2 \\
\hline $\mathrm{pCi} / \mathrm{L}$ & $\begin{array}{l}3.27 E+01 \\
\text { No flag } \\
\text { No flag } \\
\text { No flag }\end{array}$ & $\begin{array}{l}6.54 \mathrm{E}+01 \\
\text { No flag } \\
\text { No flag } \\
\text { No flag }\end{array}$ \\
\hline $\mathrm{Ci} / \mathrm{L}$ & $1.5 E+02$ & $3 E+02$ \\
\hline pCill & $5 E+01$ & $1 E+02$ \\
\hline pCi/L & $5 E+01$ & $1 E+02$ \\
\hline $\mathrm{pCi} / \mathrm{L}$ & $2.62 E+03$ & $5.24 E+03$ \\
\hline$\mu g / L$ & 50 & 100 \\
\hline $\begin{array}{l}\mu g / L \\
\mu g / L\end{array}$ & $\begin{array}{l}1,000 \\
50\end{array}$ & $\begin{array}{l}2,000 \\
100\end{array}$ \\
\hline$\mu g / L$ & 50 & 100 \\
\hline $\mathrm{pCi} / \mathrm{L}$ & $1 E+01$ & $2 E+01$ \\
\hline $\mathrm{pCi} / \mathrm{L}$ & $1 \mathrm{E}+01$ & $2 E+01$ \\
\hline $\mathrm{pCi} / \mathrm{L}$ & $1 E+01$ & $2 E+01$ \\
\hline pCi/L & $1.5 E+02$ & $3 E+02$ \\
\hline $\mathrm{pCi} / \mathrm{L}$ & $1 E+02$ & $2 E+02$ \\
\hline pCill & $1.5 E+01$ & $3 E+01$ \\
\hline$\mu g / L$ & 50 & 100 \\
\hline$\mu g / L$ & 25 & 50 \\
\hline$\mu g / L$ & 25 & 50 \\
\hline$\mu g / L$ & 25 & 50 \\
\hline & $\begin{array}{l}\text { No flag } \\
\text { No flag } \\
\text { No flag }\end{array}$ & $\begin{array}{l}\text { No flag } \\
\text { No flag } \\
\text { No flag }\end{array}$ \\
\hline$\mu \mathrm{g} / \mathrm{L}$ & 50 & 100 \\
\hline$\mu \mathrm{g} / \mathrm{L}$ & 50 & 100 \\
\hline$\mu g / L$ & 50 & 100 \\
\hline$\mu g / L$ & 2 & 4 \\
\hline & No flag & No flag \\
\hline & $\begin{array}{l}\text { No flag } \\
\text { No flag }\end{array}$ & $\begin{array}{l}\text { No flag } \\
\text { No flag }\end{array}$ \\
\hline pCi/L & $2.33 E+02$ & $4.66 E+02$ \\
\hline$\mu \mathrm{S} / \mathrm{cm}$ & 250 & 500 \\
\hline $\mathrm{pCi} / \mathrm{L}$ & $1 E+01$ & $2 E+01$ \\
\hline pCi/L & $4 E+00$ & $8 E+00$ \\
\hline $\mathrm{pCi} / \mathrm{L}$ & $4 E+00$ & $8 E+00$ \\
\hline$\mu \mathrm{g} / \mathrm{L}$ & & 100 \\
\hline$\mu g / L$ & 200,000 & 400,001 \\
\hline$\mu g / L$ & 5,000 & 10,000 \\
\hline$\mu \mathrm{g} / \mathrm{L}$ & 50 & 100 \\
\hline & No flag & No flag \\
\hline$\mu \mathrm{g} / \mathrm{L}$ & 0.000015 & 0.00003 \\
\hline$\mu g / L$ & 0.002 & 0.004 \\
\hline pCi/L & $4.5 E+02$ & $9 E+02$ \\
\hline$\mu \mathrm{g} / \mathrm{L}$ & & 100 \\
\hline$\mu \mathrm{g} /$ & 0.00225 & 0.0045 \\
\hline$\mu \mathrm{g} / \mathrm{L}$ & 0.002 & 0.004 \\
\hline$P_{0}$ & 5 & 10 \\
\hline$\mu g$ & 5 & 10 \\
\hline$\mu g / L$ & 2.5 & 5 \\
\hline$\mu g / L$ & 50 & 100 \\
\hline$\mu \mathrm{g} / \mathrm{L}$ & 1 & 2 \\
\hline
\end{tabular}

Source $^{a}$

Proposed PDWS (EPA, 1991)

Set by EPD/EMS

Set by EPD/EMS

Set by EPD/EMS

Proposed PDWS (EPA, 1986)

EPA Method 901.1

EPA Method 901.1

Proposed PDWS (EPA, 1991)

EPA Method 8270

EPA Method 8240

EPA Method 8270

EPA Method 8270

Proposed PDWS (EPA, 1991)

Proposed PDWS (EPA, 1991)

Proposed PDWS (EPA, 1991)

Proposed PDWS (EPA, 1991)

Interim Final PDWS (EPA, 1977)

Interim Final PDWS (EPA, 1977)

EPA Method 8270

Final PDWS (EPA, 1993a)

Final PDWS (EPA, 1993a)

Final PDWS (EPA, 1993a)

Set by EPD/EMS

Set by EPD/EMS

Set by EPD/EMS

SDWS (EPA, 1993b)

SDWS (EPA, 1993b)

SDWS (EPA, 1993b)

Final PDWS (EPA, 1993a)

Set by EPD/EMS

Set by EPD/EMS

Set by EPD/EMS

Proposed PDWS (EPA, 1991)

Set by EPD/EMS

Interim Final PDWS (EPA, 1977)

Final PDWS (EPA, 1993a)

Final PDWS (EPA, 1993a)

Final PDWS (EPA, 1993a)

Proposed PDWS (EPA, 1990)

EPA Method 9030

EPA Method 8270

Set by EPD/EMS

Final PDWS (EPA, 1993a)

EPA Method 8280

Interim Final PDWS (EPA, 1977)

EPA Method 8270

EPA Method 8280

EPA Method 8280

EPA Method 8240

EPA Method 8240

Final PDWS (EPA, 1993a)

EPA Method 8270

Final PDWS (EPA, 1993a) 
Analyte

Thallium, dissolved

Thallium, total recoverable

Thionazin

Thorium-228

Thorium-230

Thorium-232

Thorium-234

Tin

Tin, dissolved

Tin, total recoverable

Tin-113

Toluene

o-Toluidine

Total carbon

Total coliform

Total dissolved solids

Total hydrocarbons

Total inorganic carbon

Total organic carbon

Total organic halogens

Total organic nitrogen

Total petroleum hydrocarbons

Total phosphates (as P)

Total phosphorus

Toxaphene

2,4,5-TP (Silvex)

Tributyl phosphate

1,2,4-Trichlorobenzene

1,1,1-Trichloroethane

1,1,2-Trichloroethane

Trichloroethylene

Trichlorofluoromethane

2,4,5-Trichlorophenol

2,4,6-Trichlorophenol

2,4,5-Trichlorophenoxyacetic acid

1,2,3-Trichloropropane

$0,0,0$-Triethyl phosphorothioate

1,3,5-Trinitrobenzene

Tritium

Turbidity ${ }^{g}$

Uranium

Uranium, dissolved

Uranium, total recoverable

Uranium alpha activity

Uranium-233/234

Uranium-234

Uranium-235

Uranium-238

Vanadium

Vanadium, dissolved

Vanadium, total recoverable

Vinyl acetate

\begin{tabular}{|c|c|c|c|}
\hline Unit & Flag 1 & Flag 2 & Source $^{a}$ \\
\hline$\mu g / L$ & 1 & 2 & Final PDWS (EPA, 1993a) \\
\hline$\mu \mathrm{g} / \mathrm{L}$ & 1 & 2 & Final PDWS (EPA, 1993a) \\
\hline$g / L$ & 50 & 100 & EPA Method 8270 \\
\hline $\mathrm{B} / \mathrm{i} / \mathrm{L}$ & $6.25 \mathrm{E}+01$ & $1.25 \mathrm{E}+02$ & Proposed PDWS (EPA, 1991) \\
\hline $\mathrm{Ci} / \mathrm{L}$ & $3.96 \mathrm{E}+01$ & $7.92 E+01$ & Proposed PDWS (EPA, 1991) \\
\hline $\mathrm{Ci} / \mathrm{L}$ & $4.4 \mathrm{E}+01$ & $8.8 E+01$ & Proposed PDWS (EPA, 1991) \\
\hline ill & $2 E+02$ & $4.01 \mathrm{E}+02$ & Proposed PDWS (EPA, 1991) \\
\hline I/L & 10 & 20 & EPA Method 282.2 \\
\hline / $/ \mathrm{L}$ & 10 & 20 & EPA Method 282.2 \\
\hline g/L & 10 & 20 & EPA Method 282.2 \\
\hline iill & $1.5 E+02$ & $3 E+02$ & Interim Final PDWS (EPA, 1977) \\
\hline & 500 & 1,000 & Final PDWS (EPA, 1993a) \\
\hline$M L$ & 50 & 100 & EPA Method 8270 \\
\hline /L & 5,000 & 10,000 & EPA Method 9060 \\
\hline & & & Final PDWS (EPA, 1993a) \\
\hline & No flag & No flag & Set by EPD/EMS \\
\hline 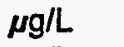 & 5,000 & 10,000 & EPA Method 418.1 \\
\hline 3/L & 5,000 & 10,000 & EPA Method 9060 \\
\hline$g / L$ & 5,000 & 10,000 & EPA Method 9060 \\
\hline  & 25 & 50 & EPA Method 9020 \\
\hline $\boldsymbol{g} \mathbf{L}$ & 500 & 1,000 & APHA Method 420 \\
\hline$g / L$ & 5,000 & 10,000 & EPA Method 418.1 \\
\hline & No flag & No flag & Set by EPD/EMS \\
\hline & No flag & No flag & Set by EPD/EMS \\
\hline IL & 1.5 & 3 & Final PDWS (EPA, 1993a) \\
\hline L & 25 & 50 & Final PDWS (EPA, 1993a) \\
\hline glL & 50 & 100 & EPA Method 8270 \\
\hline$g / L$ & 35 & 70 & Final PDWS (EPA, 1993a) \\
\hline$\mu g / L$ & 100 & 200 & Final PDWS (EPA, 1993a) \\
\hline 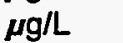 & 2.5 & 5 & Final PDWS (EPA, 1993a) \\
\hline$g / L$ & 2.5 & 5 & Final PDWS (EPA, 1993a) \\
\hline$u g / L$ & 5 & 10 & EPA Method 8240 \\
\hline al & 50 & 100 & EPA Method 8270 \\
\hline /L & 50 & 100 & EPA Method 8270 \\
\hline /L & 2.5 & 5 & EPA Method 8150 \\
\hline$\mu g / L$ & 5 & 10 & EPA Method 8240 \\
\hline 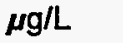 & 50 & 100 & EPA Method 8270 \\
\hline 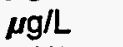 & 50 & 100 & EPA Method 8270 \\
\hline i & $1 E+01$ & $2 E+01$ & Final PDWS (EPA, 1993a) \\
\hline & No flag & No flag & Set by EPD/EMS \\
\hline O & 10 & 20 & Proposed PDWS (EPA, 1991) \\
\hline & 10 & 20 & Proposed PDWS (EPA, 1991) \\
\hline$\mu \mathrm{g} / \mathrm{L}$ & 10 & 20 & Proposed PDWS (EPA, 1991) \\
\hline $\mathrm{pCi} / \mathrm{L}$ & $1.5 \mathrm{E}+01$ & $3 E+01$ & Proposed PDWS (EPA, 1991) \\
\hline pCill & $6.9 \mathrm{E}+00$ & $1.38 E+01$ & Proposed PDWS (EPA, 1991) \\
\hline DCill & $6.95 \mathrm{E}+00$ & $1.39 E+01$ & Proposed PDWS (EPA, 1991) \\
\hline ii/L & $7.25 E+00$ & $1.45 E+01$ & Proposed PDWS (EPA, 1991) \\
\hline $\mathrm{pCi} / \mathrm{L}$ & $7.3 E+00$ & $1.46 E+01$ & Proposed PDWS (EPA, 1991) \\
\hline & 40 & 80 & EPA Method 6010 \\
\hline & 40 & 80 & EPA Method 6010 \\
\hline 8 & 40 & 80 & EPA Method 6010 \\
\hline & 5 & 10 & EPA Method 8240 \\
\hline
\end{tabular}




\begin{tabular}{|c|c|c|c|c|}
\hline Analyte & Unit & Flag 1 & Flag 2 & Source $^{a}$ \\
\hline Xylenes & $\mu g / L$ & 5,000 & 10,000 & Final PDWS (EPA, 1993a) \\
\hline Yttrium-88 & pCill & $5 E+01$ & $1 E+02$ & EPA Method 901.1 \\
\hline Zinc & $\mu \mathrm{g} / \mathrm{L}$ & 2,500 & 5,000 & SDWS (EPA, 1993b) \\
\hline Zinc, dissolved & $\mathrm{\mu g} / \mathrm{L}$ & 2,500 & 5,000 & SDWS (EPA, 1993b) \\
\hline Zinc, total recoverable & $\mu \mathrm{gg} / \mathrm{h}$ & 2,500 & 5,000 & SDWS (EPA, 1993b) \\
\hline Zinc-65 & pCi/L & $1.5 E+02$ & $3 E+02$ & Interim Final PDWS (EPA, 1977) \\
\hline Zirconium-95 & $\mathrm{pCi} / \mathrm{L}$ & $1 E+02$ & $2 \mathrm{E}+02$ & Interim Final PDWS (EPA, 1977) \\
\hline Zirconium/Niobium-95 ${ }^{c}$ & $\mathrm{pCi} / \mathrm{L}$ & $1 E+02$ & $2 E+02$ & Interim Final PDWS (EPA, 1977) \\
\hline
\end{tabular}

a References for methods are in Appendix E; references for dated sources are at the end of this appendix.

b EMS is currently unable to perform this analysis.

c EMS discontinued monitoring this radionuclide because it is inappropriate for the SRS Groundwater Monitoring Program.

d EPD/EMS set this flagging criterion using the 1991 proposed PDWS because the final PDWS in 1977 may have been in error.

e For double radionuclide analyses where each separate radionuclide has its own standard, the more stringent standard is used.

f The applied standard is for radium-226.

g The primary maximum contaminant level range for turbidity is $1-5$ NTU, which is inappropriate for the SRS Groundwater Monitoring Program.

\section{References Cited}

EPA (U.S. Environmental Protection Agency), 1977. National Interim Primary Drinking Water Regulations, EPA-570/9-76-003. Washington, DC.

EPA (U.S. Environmental Protection Agency), 1986. Water Pollution Control; National Primary Drinking Water Regulations, Radionuclides (Proposed). Federal Register, September 30, 1986, pp. 34835-34862. Washington, DC.

EPA (U.S. Environmental Protection Agency), 1990. National Primary and Secondary Drinking Water Regulations; Synthetic Organic Chemicals and Inorganic Chemicals (Proposed Rule). Federal Register, July 25, 1990 , pp. 30369-30448. Washington, DC.

EPA (U.S. Environmental Protection Agency), 1991. National Primary Drinking Water Regulations; Radionuclides; Proposed Rule. Federal Register, July 18, 1991, pp. 33052-33127. Washington, DC.

EPA (U.S. Environmental Protection Agency), 1993a. National Primary Drinking Water Regulations. Code of Federal Regulations, Title 40, Part 141, pp. 592-732. Washington, DC.

EPA (U.S. Environmental Protection Agency), 1993b. National Secondary Drinking Water Regulations. Code of Federal Regulations, Title 40, Part 143, pp. 774-777. Washington, DC.

SCDHEC (South Carolina Department of Health and Environmental Control), 1981. State Primary Drinking Water Regulations, R.61-58.5. Columbia, SC. 
WSRC-TR-96-0054

Unclassified

\section{Appendix C}

Figures 
WSRC-TR-96-0054

Unclassified

THIS PAGE LEFT BLANK INTENTIONALLY. 


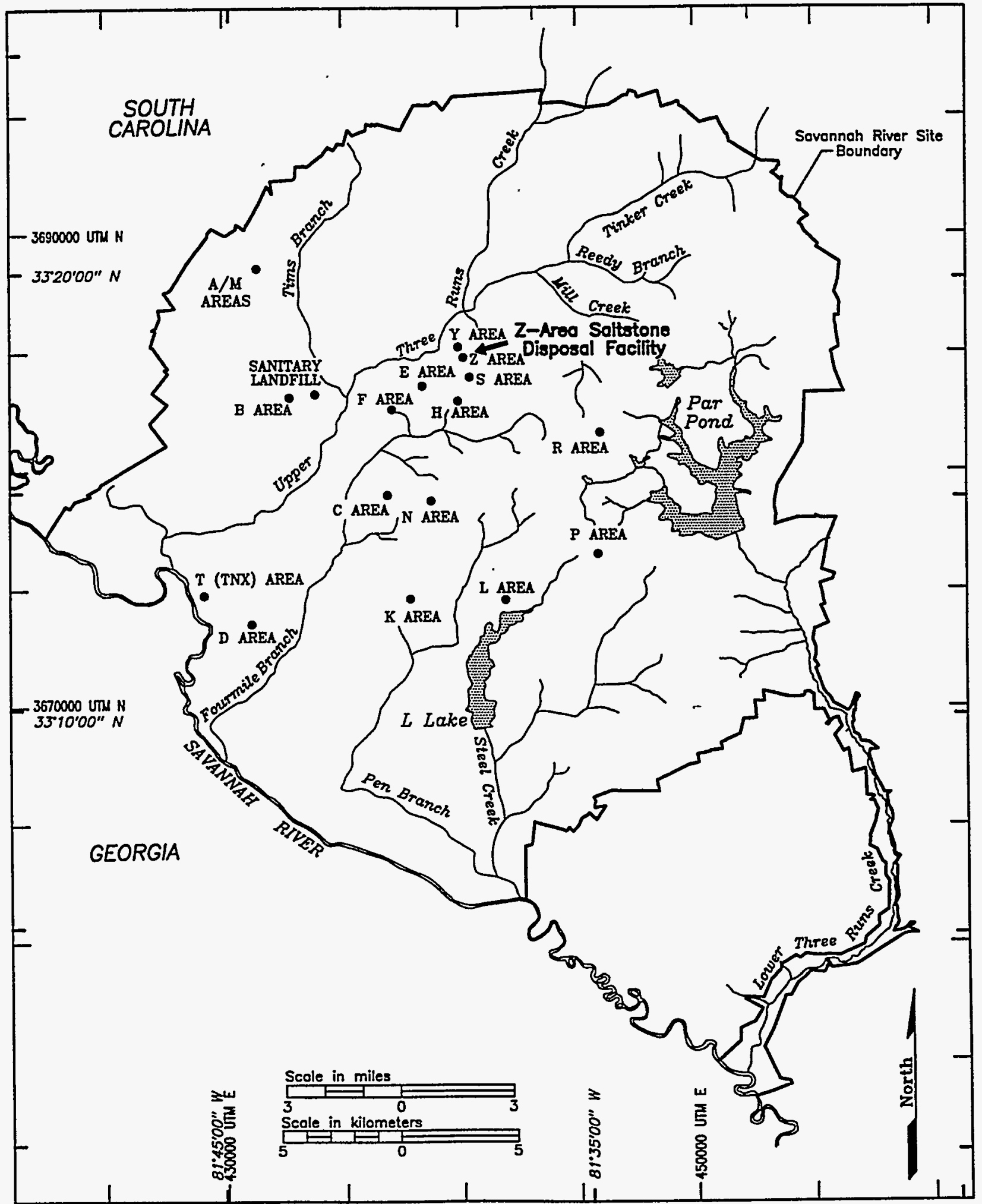

Figure 1. Location of the Z-Area Saltstone Disposal Facility at the Savannah River Site 


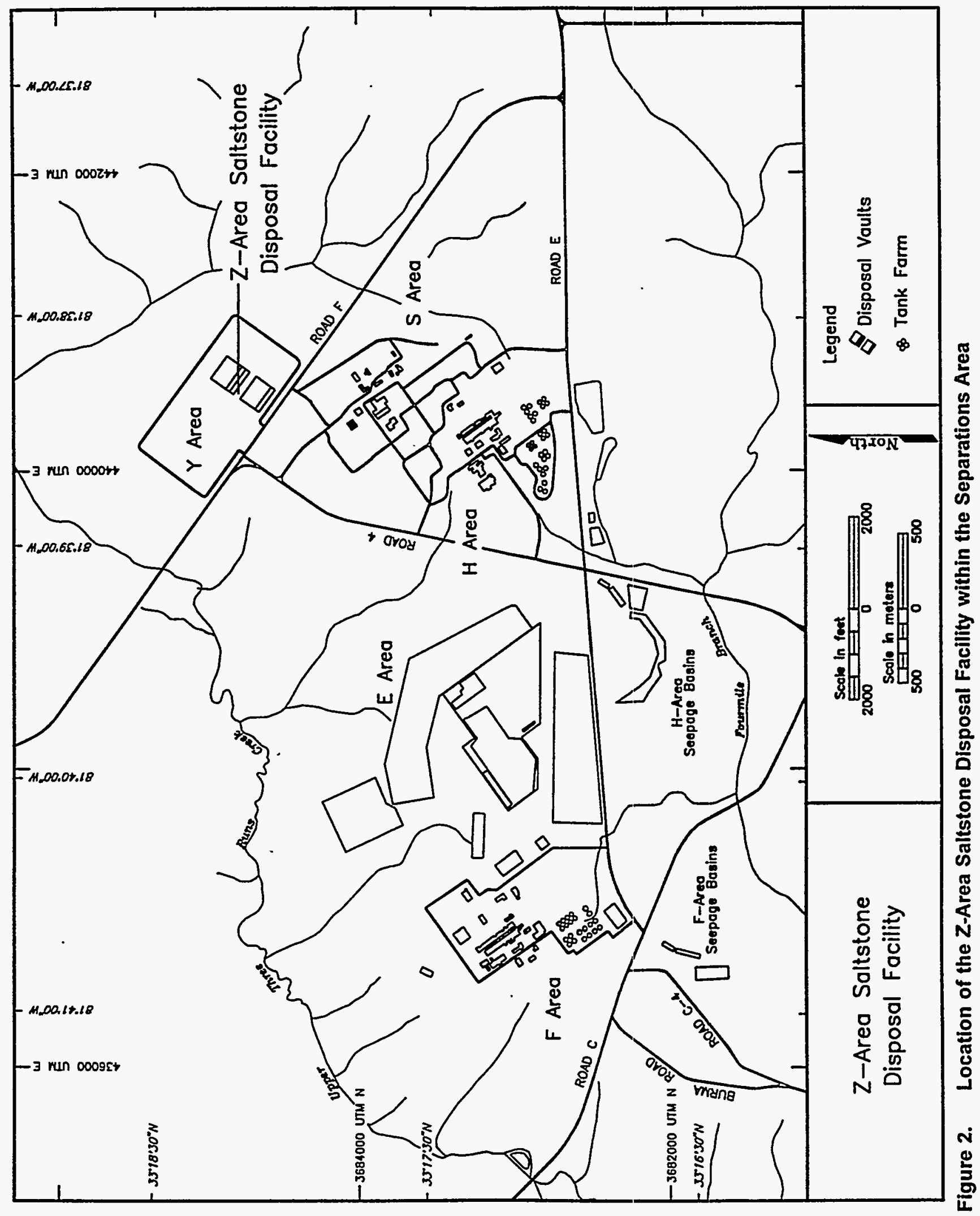




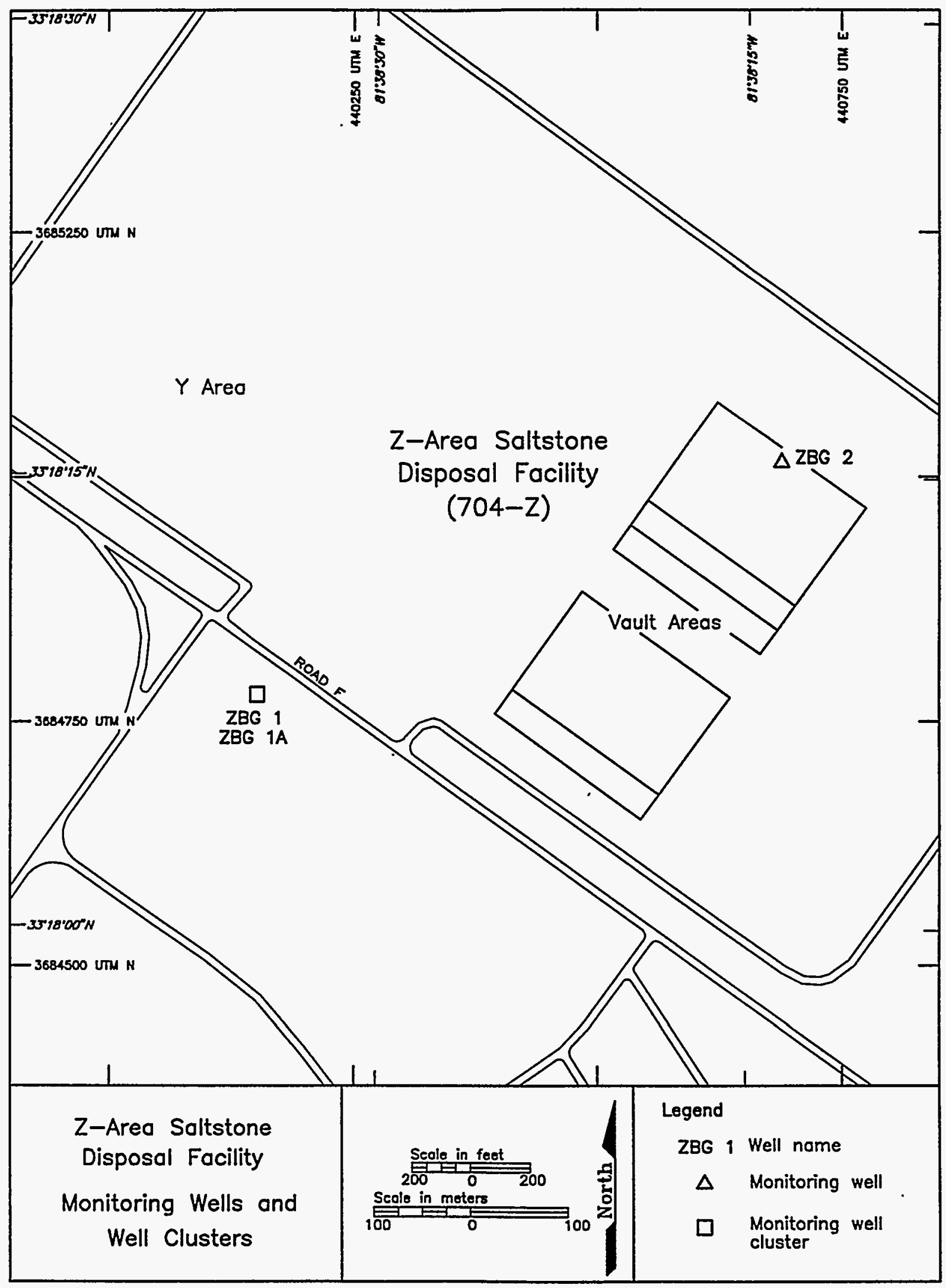

Figure 3. Location of Groundwater Monitoring Wells at the Z-Area Saltstone Disposal Facility 
WSRC-TR-96-0054

Unclassified

THIS PAGE LEFT BLANK INTENTIONALLY. 


\section{Appendix D}

\section{Groundwater Monitoring Results Tables}


WSRC-TR-96-0054

Unclassified

THIS PAGE LEFT BLANK INTENTIONALLY. 


\section{Key to Reading the Tables}

The following abbreviations may appear in the data tables:

\section{Constituents}

\section{$1,2,3,4,6,7,8-\mathrm{HPCDD}$ \\ $1,2,3,4,6,7,8-\mathrm{HPCDF}$ \\ $1,2,3,4,7,8-H X C D D$ \\ $1,2,3,4,7,8-\mathrm{HXCDF}$ \\ Lindane \\ PCB}

1,2,3,7,8-PCDD

$1,2,3,7,8-P C D F$

Sp. conductance

TCDD

TCDF

\section{Laboratories}

\section{CN \\ EM}

GE and GP

SC

SP

TM

WA and WS

\section{Sampling Codes}

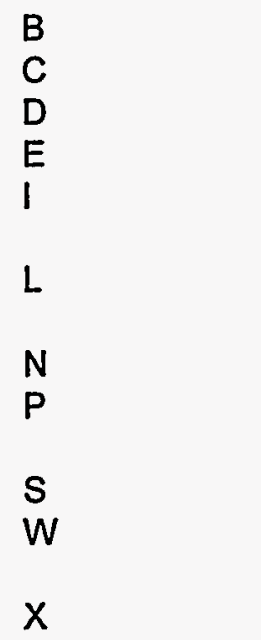

Sampling Methods

B

P $1,2,3,4,6,7,8$-heptachlorodibenzo-p-dioxin

$1,2,3,4,6,7,8$-heptachlorodibenzo-p-furan

$1,2,3,4,7,8$-hexachlorodibenzo-p-dioxin

1,2,3,4,7,8-hexachlorodibenzo-p-furan

gamma-benzene hexachloride

polychlorinated biphenyl

$1,2,3,7,8$-pentachlorodibenzo-p-dioxin

1,2,3,7,8-pentachlorodibenzo-p-furan

specific conductance

tetrachlorodibenzo-p-dioxin

tetrachlorodibenzo-p-furan

Clemson Technical Center, Inc.

Environmental Protection Department/Environmental

Monitoring Section (EPD/EMS) Laboratory

General Engineering Laboratories, Inc.

Savannah River Technology Center

Spencer Testing Services, Inc.

TMAVEberline

Roy F. Weston, Inc.

blank sample was collected

well was pumping continuously

well was dry

equipment blank was collected

well went dry during sampling; insufficient water to collect

all samples

well went dry before sampling began; only depth to water

can be determined

well was not stabilized before sampling began

inaccessibility or mechanical failure prevented sample

collection and field analysis of the water

no water in standpipe; for water-level events only

purged water must be containerized; only water-level

measurements were obtained

well went dry during purging; samples collected after well recovered

sample collected using an open-bucket bailer

sample collected using a bladder pump 
sample collected using a single-speed centrifugal

downhole pump

V sample collected using a variable-speed pump

Units

$\mathrm{mg} / \mathrm{L}$

milligrams per liter

msI

MSL

mean sea level

NTU

$\mathrm{pCi} / \mathrm{L}$

$\mathrm{pCi} / \mathrm{mL}$

$\mathrm{pH}$

$\mu \mathrm{g} / \mathrm{L}$

$\mu \mathrm{S} / \mathrm{cm}$

million structures per liter

nephelometric turbidity unit

picocuries per liter

picocuries per milliliter

$\mathrm{pH}$ unit

micrograms per liter

microsiemens per centimeter

Other

$\begin{array}{ll}\text { CS } & \text { carbon steel } \\ \text { DF } & \text { dilution factor column in data tables } \\ \text { E } & \begin{array}{l}\text { exponential notation }\left(e .9 ., 1.1 E-09=1.1 \times 10^{-9}=\right. \\ \text { holding time column in data tables }\end{array} \\ \text { H } & \text { modifier column in data tables } \\ \text { Mod } & \text { primary drinking water standard } \\ \text { PDWS } & \text { polyvinyl chloride } \\ \text { PVC } & \text { exceeded the final PDWS or screening level column in } \\ \text { ST } & \text { data tables } \\ \text { TOC } & \text { top of casing }\end{array}$

\section{Holding Times}

Standard analytical methods include a limit, called holding time, on the maximum elapsed time between sample collection and extraction or analysis by the laboratory. In the data tables, a large bullet $(\cdot)$ in the $H$ (holding time) column indicates that holding time was exceeded. Analyses performed beyond holding times may not yield valid results.

The South Carolina Department of Health and Environmental Control (SCDHEC) allows only 15 minutes to elapse between sampling and analysis for $\mathrm{pH}$. Thus, only field $\mathrm{pH}$ measurements can meet the holding time criterion; laboratory $\mathrm{pH}$ analyses always will exceed it.

The laboratory procedure used for the determination of specific conductance allows one day to elapse between sampling and analysis. Thus, laboratory specific conductance measurements may exceed the holding time criterion.

\section{Data Rounding}

Constituent results in analytical results tables that appear to equal the final PDWS but are not marked in the ST (exceeded the final PDWS or screening level) column are below the final PDWS in the database. Values stored in the database contain more significant digits than the reported results. Apparent discrepancies in the tables are due to the rounding of reported results. 


\section{Data Qualification}

The contract laboratories submit sample- or batch-specific quality assurance/quality control information either at the same time as analytical results or in a quarterly summary. Properly defined and used data modifiers (also referred to as qualifiers) can be a key component in assessing data usability. Modifiers designed by EPD/EMS and provided to the primary laboratories are defined below. These modifiers appear in the data tables under the column Mod. The lettered modifiers are based on EPA's STORET codes.

\section{Modifiers}

Data are not qualified. Numbers should be interpreted exactly as reported.

1

$J$

L

M

$\mathbf{R}$

$\mathrm{T}$

U

V or W

Y

1

2

3
The value in the result field is the instrument reading, not the sample quantitation limit. Always used with the result qualifier $U$.

Value is estimated because quantitation in the sample or in associated quality control samples did not meet specifications.

Value is off-scale high. The actual value is not known but is known to be greater than the value shown.

Presence of the analyte is verified but not quantified.

Result was rejected because performance requirements in the sample analysis or associated quality control analyses were not met.

Analyte was not detected; if present, it was below the criteria for detection.

Material analyzed for but not detected. Analytical result reported is less than the sample quantitation limit.

Analyte was detected in an associated method blank.

Result was obtained from an unpreserved or improperly preserved sample. Data may not be accurate.

Result may be an underestimation of the true value due to analytical bias.

Result may be an overestimation of the true value due to analytical bias.

The associated result may be of poor precision (high variability) due to analytical bias.

Note: These are only some of the qualifiers present in the database. All modifiers associated with the data are published in the result tables of EPD/EMS' quarterly groundwater monitoring reports, the official repository of the data. 
Table 1. Maximum Levels of Constituents Exceeding the South Carolina Department of Health and Environmental Control Proposed Groundwater Monitoring Standards or the Final Primary Drinking Water Standards

\begin{tabular}{lccccccc}
\hline Well & Constituent & Unit & 1Q95 & 2Q95 & 3Q95 & 4Q95 & Mod \\
\hline Aquifer Zone IIB2 (Water Table) & & & & & & & \\
ZBG 1 & Dichloromethane & $\mu \mathrm{g} / \mathrm{L}$ & - & 9.3 & - & - \\
\hline
\end{tabular}

Table 2. Maximum Levels of Constituents Exceeding Half the Final Primary Drinking Water Standards or Other SRS Flagging Criteria

\begin{tabular}{llllll}
\hline Well & Constituent & Unit & 4Q95 & Flag & Mod \\
\hline N & None & N & N & N & N \\
\hline
\end{tabular}

$\mathrm{N}=$ not applicable.

$-=$ analyzed but not above the GWPS.

Notes: This table presents the highest value for duplicate/replicate results. The groundwater samples are unfiltered. Therefore, the results for the metals are for total recoverable metals. Flags are established by EPD/EMS and are based on final PDWS, Secondary Drinking Water Stanclards, or method detection limits (see Appendix B). 
Table 3. Groundwater Monitoring Results for Individual Wells

WELL ZBG 1

\begin{tabular}{|c|c|}
\hline SRS Coord. & Lat/Longitude \\
\hline $\begin{array}{l}\text { N76584.2 } \\
\text { E65584.1 }\end{array}$ & $\begin{array}{l}33.3021611^{\circ} \mathrm{N} \\
81.642863^{\circ} \mathrm{W}\end{array}$ \\
\hline
\end{tabular}

SAMPLE DATE

FIELD DATA

\author{
Screen Zone Elevation \\ 240.1-220.0 ft msl
}

$02 / 16 / 95$

$04 / 17 / 95$

$\begin{array}{ll}\text { Casing } & \text { Pump } \\ \text { 4" PVC } & \text { S }\end{array}$

Screen Zone

Water Table

Analyte
Water elevation
Depth to water
pH
Sp. conductance
Water temperature
Alkalinity as CaCO
Turbidity
Volume purged
Sampling code

ANALYTICAL DATA

H ST Analvte

Actinium-228

Aluminum, total recoverable

Antimony, total recoverable

Antimony-124

Antimony-125

Arsenic, total recoverable

Barium, total recoverable

Barium-133

Benzene

Bromodichloromethane

Bromoform

Bromomethane (Methyl bromide)

Cadmium, total recoverable

Carbon tetrachloride

Cerium-144

Cesium-134

Cesium-137

Chlorobenzene

Chlorosthane

Chloroethene (Vinyl chloride)

2-Chloroethyl vinyl ether

Chloroform

Chloromethane (Methyl chloride)

Chromium, total recoverable

Cobalt-57

Cobalt-58

Cobalt -60

Dibromochloromethane

1.1-Dichloroethane

1,2-Dichloroethane

1,1-Dichloroethylene

1,2-Dichloroethylene

trans-1,2-Dichloroethylene

Dichloromethane

1,2-Dichloropropane

cis-1,3-Dichloropropene

trans-1,3-Dichloropropene

Ethylbenzene

Europium-152

Europium-154

1095
233.9
57.2
5.5
22
19.9
1
1
6.3

2095
234.9
56.2
5.2
24
20.2
1
2
4.5

\section{5}

$3.5 \mathrm{E}+00$

$<33$

$<3.3$

3.5E-01
$-2.6 \mathrm{E}-01$

$<3.3$

9.3

$<1.7$ + 1.70

$<1.7$

$<1.7$

$<1.7$

$<3.3$

$<1.7$

$-4.4 E-01$

$1.6 E+00$

$<1.7$

$<1.7$

$<1.7$

$<1.7$

$<1.7$

$<1.7$

$<6.7$

6.1E-02

$-1.7 E-01$

$-2.7 E-01$

$<1.7$
$<1.7$

$<1.7$

$<1.7$

$<1.7$
$<1.7$

$<1.7$

$<1.7$

$<1.7$

$<1.7$

$<1.7$

9.5E-01

$4.2 E+00$ $\underline{2095}$

$-3.7 E+00$

3095

$3.8 E+\infty 0$

$<5.0$

$-3.1 E+\infty 0$

$-4.4 E+00$

8.4

$-2.9 E+00$

$<1.7$

$<1.7$

$<1.7$

$<1.7$

0.58

$<1.7$

$-1.8 E+00$

$-1.6 \mathrm{E}+00$

$-1.2 E+00$

$<1.7$

$<1.7$

$<1.7$

$<1.7$

$<1.7$

$<1.7$
$<1.7$

$<1.7$
$<4.0$

$-2.1 E+00$

$-1.5 E+00$

$-2.5 E+00$

$<1.7$

$<1.7$

$<1.7$

$<1.7$

$<1.7$
$<5.0$

$<1.7$

9.3
$<1.7$

$<1.7$

$<1.7$

$<1.7$

$<1.7$

$-8.9 E+00$

$-1.2 E+00$
$<5.0$

$-2.2 E-02$

8.6E-01

$8.6 \mathrm{E}$
$<5.0$
7.9

$1.1 E+00$

$<2.0$

$<2.0$

$<2.0$

$<2.0$

$<2.0$

1. $6 \mathrm{E}+00$

8.5E-01

$-4.5 E-01$

$<2.0$

$<2.0$

$<2.0$

$<2.0$

$<2.0$

$<4.0$

$-1.1 E+00$

-2.3E-01

$-1.8 \mathrm{E}-01$

$<2.0$
$<2.0$

$<2.0$

$<2.0$

$<2.0$

$<2.0$
$<2.0$

$<2.0$

$<2.0$

$<2.0$

$-1.4 \mathrm{E}+00$

$-9.9 E+00$

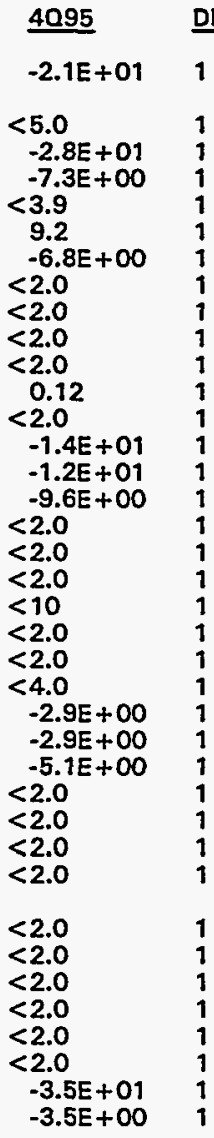

Unit

ft msl

$\mathrm{ft}$

$\mu \$ / \mathrm{cm}$

${ }^{\circ} \mathrm{C}$

mg/L

well vol.

Note: Flagging levels, modifiers, and laboratories are for 4th quarter 1995 data only. See Appendix B for flagging criteria.

- = exceeded holding time for 4th quarter 1995.

a = exceeded SCDHEC proposed groundwater monitoring standard, final primary drinking water standard, or screening level for 4th quarter 1995. 


\section{ANALYTICAL DATA}

\section{H ST Analyte}

Europium-155
Gross alpha
lodine-129
Lead, total recoverable
Lead-212
Manganese-54
Mercury, total recoverable
Neptunium-239
Nitrate as nitrogen
Nitrate-nitrite as nitrogen
Nitrite as nitrogen
Potassium-40
Promethium-144
Promethium-146
Radium, total alpha-emitting
Ruthenium-106
Selenium, total recoverable
Silver, total recoverable
Sodium-22
Technetium-99
1,1,2,2-Tetrachloroethane
Tetrachloroethylene
Thorium-234
Tin-113
Toluene
Total organic carbon
1,1-Trichloroethane
1,1,2-Trichlorothane
Trichloroethylene
Trichlorofluoromethane
Tritium
Xylenes
Ytrium-88
Zinc-65
Zirconium-95

\begin{tabular}{|c|c|c|c|c|c|c|c|c|}
\hline 1095 & $\underline{2095}$ & 3095 & 4095 & DF & Mod & Unit & Lab & Flag \\
\hline $\begin{array}{l}4.0 E+00 \\
1.6 E+00 \\
5.6 \\
2.2 E+00 \\
-6.6 E-01 \\
<0.33 \\
-8.5 E+00\end{array}$ & $\begin{array}{c}-1.4 E+01 \\
1.7 E+00 \\
-1.5 E+01 \\
9.8 \\
1.0 E+00 \\
-5.4 E-01 \\
<0.20 \\
-9.1 E+00 \\
1.460\end{array}$ & $\begin{array}{l}1.8 E+\infty 0 \\
1.1 E+\infty 0 \\
3.2 \\
3.7 E+\infty 0 \\
-1.0 E+\infty \\
<0.20 \\
6.8 E+\infty\end{array}$ & $\begin{array}{l}-4.5 E+01 \\
1.7 E+00 \\
-6.5 E+02 \\
4.5 \\
-1.4 E+01 \\
4.0 E-02 \\
0.024 \\
-9.1 E+01\end{array}$ & $\begin{array}{l}1 \\
1 \\
1 \\
1 \\
1 \\
1 \\
1 \\
1\end{array}$ & $\begin{array}{l}\text { UI } \\
\text { UI } \\
J \\
\text { UI } \\
\text { UI } \\
J \\
\text { UI }\end{array}$ & $\begin{array}{l}\mathrm{pCi} / \mathrm{L} \\
\mathrm{pCi} / \mathrm{L} \\
\mathrm{pCi} / \mathrm{L} \\
\mu \mathrm{g} / \mathrm{L} \\
\mathrm{pCi} / \mathrm{L} \\
\mathrm{pCi} / \mathrm{L} \\
\mu \mathrm{g} / \mathrm{L} \\
\mathrm{pCi} / \mathrm{L} \\
\mu \mathrm{g} / \mathrm{L}\end{array}$ & $\begin{array}{l}\mathrm{TM} \\
\mathrm{TM} \\
\mathrm{TM} \\
\mathrm{GE} \\
\mathrm{TM} \\
\mathrm{TM} \\
\mathrm{GE} \\
\mathrm{TM}\end{array}$ & $\begin{array}{l}0 \\
0 \\
0 \\
0 \\
0 \\
0 \\
0 \\
0\end{array}$ \\
\hline $\begin{array}{c}1,120 \\
<5.0 \\
2.1 E+01 \\
-1.4 E-01 \\
-5.0 E-01 \\
5.0 E-01 \\
-3.8 E+00 \\
<3.3 \\
<3.3 \\
6.0 E-01 \\
-2.4 E+01 \\
<1.7 \\
<1.7 \\
6.3 E+00 \\
-5.7 E-01 \\
<1.7 \\
<1.670\end{array}$ & $\begin{array}{l}1,200 \\
2.0 \\
5.0 E+00 \\
-5.7 E-01 \\
-2.7 E+00 \\
1.0 E+00 \\
-1.7 E+01 \\
2.4 \\
0.48 \\
-5.1 E-01 \\
<-2.5 E+01 \\
<1.7 \\
<1.7 \\
1.6 E+02 \\
-9.1 E-01 \\
<1.7\end{array}$ & $\begin{array}{c}1,100 \\
<5.0 \\
3.6 \mathrm{E}+\infty 0 \\
-2.3 \mathrm{E}-01 \\
2.0 \mathrm{E}-01 \\
4.0 \mathrm{E}-01 \\
-1.1 \mathrm{E}+00 \\
<5.0 \\
<2.0 \\
5.0 \mathrm{E}-01 \\
-8.2 \mathrm{E}+00 \\
<10 \\
<2.0 \\
3.7 \mathrm{E}+00 \\
-2.8 \mathrm{E}-01 \\
<2.0\end{array}$ & $\begin{aligned} & 1.100 \\
&< 5.0 \\
&-4.2 E+01 \\
&-1.4 E+00 \\
&-2.5 E+00 \\
& 1.0 E+00 \\
&-3.1 E+01 \\
&<5.0 \\
&<2.0 \\
&-1.4 E+00 \\
& 7.6 E-01 \\
&<2.0 \\
&<2.0 \\
&-5.0 E+02 \\
&-2.8 E+00 \\
&<2.0\end{aligned}$ & $\begin{array}{l}1 \\
1 \\
1 \\
1 \\
1 \\
1 \\
1 \\
1 \\
1 \\
1 \\
1 \\
1 \\
1 \\
1 \\
1 \\
1\end{array}$ & $\begin{array}{l}J \\
\text { UI } \\
U I \\
\text { UI } \\
J \\
U I\end{array}$ & 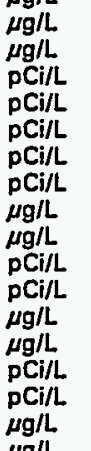 & $\begin{array}{l}\text { GE } \\
\text { GE } \\
\text { TM } \\
\text { GP } \\
\text { TM } \\
\text { TM } \\
\text { TM } \\
\text { GE } \\
\text { GE } \\
\text { GP } \\
\text { GP } \\
\text { GE } \\
\text { GE } \\
\text { TM } \\
\text { GP } \\
\text { GE }\end{array}$ & $\begin{array}{l}0 \\
0 \\
0 \\
0 \\
0 \\
0 \\
0 \\
0 \\
0 \\
0 \\
0 \\
0 \\
0 \\
0 \\
0 \\
0\end{array}$ \\
\hline $\begin{aligned}<1.7 \\
<1.7 \\
<1.7 \\
<1.7 \\
\quad 9.9 E+00\end{aligned}$ & $\begin{array}{l}<1.7 \\
<1.7 \\
<1.7 \\
<1.7 \\
9.8 E+00\end{array}$ & $\begin{array}{l}<2.0 \\
<2.0 \\
<2.0 \\
<2.0 \\
\mathbf{9 . 1 E}+\infty\end{array}$ & $\begin{array}{l}<2.0 \\
<2.0 \\
<2.0 \\
<2.0 \\
1.0 E+01\end{array}$ & $\begin{array}{l}1 \\
1 \\
1 \\
1 \\
1\end{array}$ & & $\begin{array}{l}\mu \mathrm{g} / \mathrm{L} \\
\mu \mathrm{g} / \mathrm{L} \\
\mu \mathrm{g} / \mathrm{L} \\
\mu \mathrm{g} / \mathrm{L} \\
\mathrm{pCi} / \mathrm{mL}\end{array}$ & $\begin{array}{l}\text { GE } \\
\text { GE } \\
\text { GE } \\
\text { GE } \\
\text { TM }\end{array}$ & $\begin{array}{l}0 \\
0 \\
0 \\
0 \\
1\end{array}$ \\
\hline $\begin{array}{l}5.2 E-01 \\
1.6 E+00 \\
2.5 E-01\end{array}$ & $\begin{array}{l}-1.8 E+00 \\
-1.6 E+00 \\
2.0 E-01\end{array}$ & $\begin{array}{l}-5.0 E-01 \\
5.6 E-01 \\
-1.4 E-01\end{array}$ & $\begin{array}{l}-1.4 E-01 \\
-3.7 E+00 \\
-4.3 E+00\end{array}$ & $\begin{array}{l}1 \\
1 \\
1\end{array}$ & $\begin{array}{l}\text { UI } \\
\text { UI } \\
\text { UI }\end{array}$ & $\begin{array}{l}\text { pCi/L } \\
\text { pCi/L } \\
\text { pCi } / L\end{array}$ & $\begin{array}{l}\text { GP } \\
T M \\
T M\end{array}$ & $\begin{array}{l}0 \\
0 \\
0\end{array}$ \\
\hline
\end{tabular}

\section{WELL ZBG 1A}

\begin{tabular}{|c|c|c|c|c|c|c|c|}
\hline \multirow{2}{*}{$\begin{array}{l}\text { SRS Coord. } \\
\text { N76588.5 } \\
\text { E65598.8 }\end{array}$} & \multirow{2}{*}{$\begin{array}{l}\text { Lat/Longitude } \\
33.302195^{\circ} \mathrm{N} \\
81.642833^{\circ} \mathrm{W}\end{array}$} & \multirow{2}{*}{$\frac{\text { Scroen Zone Elevation }}{281.0-276.0 \mathrm{ft} \mathrm{msl}}$} & \multicolumn{2}{|c|}{ Top of Casing } & Casing & Pump & Screen Zone \\
\hline & & & $291 \mathrm{ft} \mathrm{msl}$ & & 4" PVC & B & Perched Zone \\
\hline SAMPLE DA & & $02 / 16 / 95$ & $04 / 17 / 95$ & $07 / 28 / 95$ & $10 / 31 / 95$ & & \\
\hline \multicolumn{8}{|l|}{ FIELD DATA } \\
\hline & & 1095 & $\underline{2095}$ & 3095 & 4095 & Unit & \\
\hline \multicolumn{2}{|c|}{$\begin{array}{l}\text { Water elevation } \\
\text { Depth to water } \\
\text { pH } \\
\text { Sp. conductance } \\
\text { Water temperature } \\
\text { Alkalinity as } \mathrm{CaCO}_{3} \\
\text { Turbidity } \\
\text { Volume purged } \\
\text { Sampling codo }\end{array}$} & $\begin{array}{l}\text { Dry } \\
\text { D }\end{array}$ & $\begin{array}{l}\text { Dry } \\
\text { D }\end{array}$ & $\begin{array}{l}\text { Dry } \\
\text { D }\end{array}$ & $\begin{array}{l}\text { Dry } \\
\text { D }\end{array}$ & $\begin{array}{l}\text { ft msl } \\
\mathrm{ft} \\
\mathrm{pH} \\
\mu \mathrm{S} / \mathrm{cm} \\
{ }^{\circ} \mathrm{C} \\
\mathrm{mg} / \mathrm{L} \\
\text { NTU } \\
\text { well vol. }\end{array}$ & \\
\hline
\end{tabular}

Note: Flagging levels, modifiers, and laboratories are for 4th quarter 1995 data only. See Appendix B for flagging criteria. - = exceeded holding time for 4th quarter 1995.

$=$ = exceeded screening level or final primary drinking water standard for 4th quarter 1995. 
WELL ZBG 2

\begin{tabular}{|c|c|c|c|c|c|c|}
\hline SRS Coord. & Lat/Longitude & Screen Zone Elevation & Top of Casing & Casing & Pump & Screen Zone \\
\hline $\begin{array}{l}\text { N76170.5 } \\
\text { E67472.9 }\end{array}$ & $\begin{array}{l}33.304327{ }^{\circ} \mathrm{N} \\
81.637084\end{array}$ & $230.9-210.9 \mathrm{ft} \mathrm{msl}$ & $278 \mathrm{ft} \mathrm{msl}$ & 4" PVC & $\mathbf{s}$ & Water Table \\
\hline SAMPLE DATE & & $02 / 16 / 95$ & $04 / 17 / 95$ & & & \\
\hline
\end{tabular}

FIELD DATA

Analyte

Water elevation

Depth to water

$\mathrm{pH}$

$\mathrm{Sp}$. conductance

Water temperature

Alkalinity as $\mathrm{CaCO}_{3}$

Turbidity

Volume purged

Sampling code

ANALYTICAL DATA

H ST Analyte

Actinium-228

Aluminum, total recoverable

Antimony, total recoverable

Antimony-124

Antimony-125

Arsenic, total recoverable

Barium, total recoverable

Barium-133

Benzene

Bromodichloromethane

Bromoform

Bromomethane (Methyl bromide)

Cadmium, total recoverable

Carbon tetrachloride

Cerium-144

Cesium-134

Cesium-137

Chlorobenzene

Chloroethane

Chloroethene (Vinyl chloride)

2-Chloroethyl vinyl ether

Chloroform

Chloromethane (Methyl chloride)

Chromium, total recoverable

Cobalt-57

Cobalt-58

Cobalt-60

Dibromochloromethane

1.1-Dichloroethane

1,2-Dichloroethane

1,1-Dichloroethylene

trans-1,2-Dichloroethylene

Dichloromethane

1,2-Dichloropropane

cis-1,3-Dichloropropene

trans-1,3-Dichloropropene

Ethylbenzene

Europium-152

Europium-154

Europium-155

Gross alpha

Lead, total recoverable

Lead-212

Manganese, total recoverable

$\begin{array}{lll}\text { 1095 } & & \text { 2095 } \\ 222.7 & & 224.5 \\ 55.3 & & 53.5 \\ 5.2 & & 5.0 \\ 17 & & 18 \\ 20.2 & & 20.1 \\ 1 & 0 \\ 1 & 1 \\ 6.4 & 5.0\end{array}$

30.95
224.5
53.6
5.2
17
20.3
1
0
5.2

4095
224.4
53.6
4.8
18
19.7
0
1
5.8

Unit

ft msl

ft

pH

${ }^{\circ} \mathrm{C}$

$\mathrm{mg} / \mathrm{L}$

well vol.

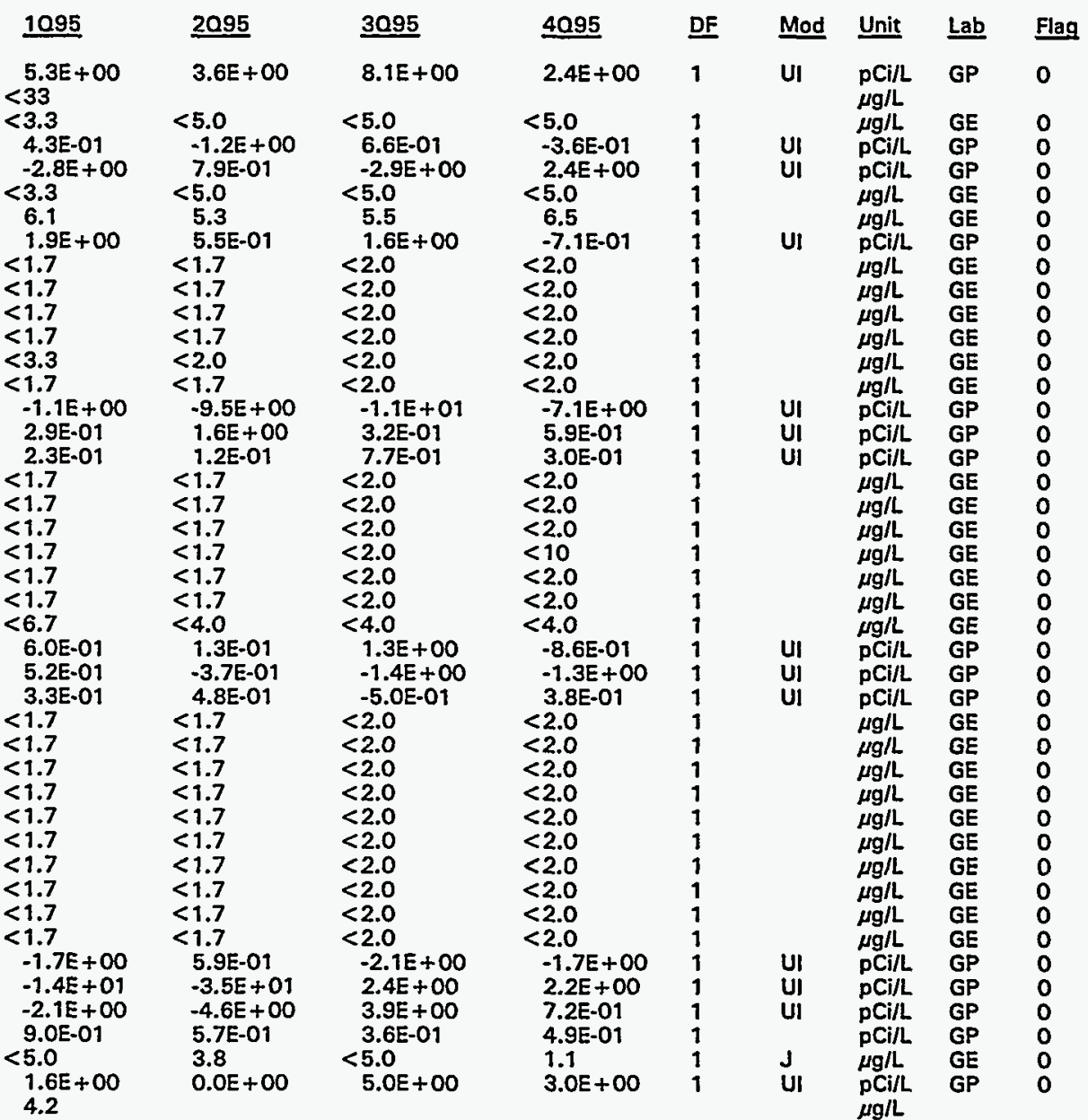

Note: Flagging levels, modifiers, and laboratories are for 4th quarter 1995 data only. See Appendix B for flagging criteria. - = exceeded holding time for 4th quarter 1995.

- = exceeded SCDHEC proposed groundwater monitoring standard, final primary drinking water standard, or screening level for 4th quarter 1995. 
H ST Analyte

Manganese-54

Mercury, total recoverable

Neptunium-239

Nickel, total recoverable

Nitrate-nitrite as nitrogen

Nitrite as nitrogen

Potassium-40

Promethium-144

Promethium-146

Radium, total alpha-emitting

Ruthenium-106

Selenium, total recoverable

Silver, total recoverable

Sodium-22

Technetium-99

1,1,2,2-Tetrachloroethane

Tetrachloroethylene

Thorium-234

Tin-113

Toluene

Total organic carbon

1,1,1-Trichloroethane

1,1,2-Trichloroethane

Trichlorosthylene

Trichlorofluoromethane

Tritium

Yttrium-88

Zinc-65

Zirconium-95

1095
$2.5 E-01$
$<0.33$
$2.7 E+00$
$<6.7$
770
$<5.0$
$7.2 E+00$
$2.6 E-02$
$4.0 E-01$
$4.0 E-01$
$-9.4 E+00$
$<3.3$
$<3.3$
$-5.3 E-01$
$-2.0 E+01$
$<1.7$
$<1.7$
$0.0 E+00$
$-1.6 E+00$
$<1.7$
$<1,670$
$<1.7$
$<1.7$
$<1.7$
$<1.7$
$8.4 E+00$
$2.1 E-01$
$-1.9 E+00$
$3.2 E+00$

\subsection{5}

3095

$-1.0 E+00$

$<0.20$

1.1E+01

2.6E-01

$<0.20$

650

$<5.0$

6.7E+00

7.1E-01

8.0E-01

$-3.0 E-01$

$-3.1 E-01$

$<5.0$

$<0.65$

$-8.5 \mathrm{E}-03$

$-2.6 \mathrm{E}+01$

$<1.7$

$<1.7$

$0.0 \mathrm{E}+00$
$-9.9 \mathrm{E}-01$

$<1.7$

760

$<5.0$

3. $3 E+01$

$2.0 E+00$

2.1E+0O

5.0E-01

$-2.5 E+00$

$<5.0$

$<2.0$

$-1.2 \mathrm{E}+00$

$-1.8 E+00$

$<10$
$<2.0$

1.2E + 02

$-1.9 E+00$

$<2.0$

$<1.7 \quad<2.0$

$<1.7$

$<1.7$

$<1.7$

$7.9 E+00$

8.3E-01

$-2.6 E+00$

$<2.0$
$<2.0$

$<2.0$

$<2.0$

$8.7 E+00$

$-1.4 E+00$

2.6E-01

4.8E-02



Note: Flagging levels, modifiers, and laboratories are for 4th quarter 1995 data only. See Appendix B for flagging criteria. - = exceeded holding time for 4th quarter 1995.

- = exceeded screening level or final primary drinking water standard for 4th quarter 1995. 


\section{Appendix E}

\section{Data Quality/Usability Assessment}


WSRC-TR-96-0054

Unclassified

THIS PAGE LEFT BLANK INTENTIONALIY. 


\section{Data Quality/Usability Assessment}

Quality assurance/quality control (QA/QC) procedures relating to accuracy and precision of analyses performed on groundwater samples are followed in the field and laboratory and are reviewed prior to publication of results. The review by the Environmental Protection Department/ Environmental Monitoring Section (EPD/EMS) of the volume of analytical data acquired each quarter and presented in various reports is an ongoing process; EPD/EMS' review of the QA/QC data cannot be completed in time to meet the deadlines for the groundwater monitoring reports required by the Resource Conservation and Recovery Act and associated regulations. Other site and regulatory personnel can obtain further information on the data quality and usability in a variety of ways, including those described below.

\section{Results below Detection}

For radiological analyses, the analytical result field contains the result recorded on the analytical instrument and reported by the laboratory, even if it is negative. For non-radiological analyses, if the analyte is not detected, the sample-specific estimated quantitative limit (EQL) is entered into the result field (with a less than $[<]$ sign). The EQL is defined as the lowest concentration that can be achieved reliably within specified limits of precision and accuracy during routine laboratory operating conditions. The sample-specific EQL is modified for sample concentration or dilution or unusual aliquot size that affects analytical sensitivity.

\section{Data Qualification}

The contract laboratories continually assess their own accuracy and precision according to U.S. Environmental Protection Agency (EPA) guidelines. They submit sample- or batch-specific QA $Q C$ information either at the same time as analytical results or in quarterly summaries. Properly defined and used modifiers (also referred to as qualifiers) can be a key component in assessing data usability. Modifiers designed by EPD/EMS and used by the primary laboratories are presented in Appendix D.

\section{Assessment of Accuracy of the Data}

Accuracy, or the nearness of the reported result to the true concentration of a constituent in a sample, can be assessed in several ways.

A laboratory's general accuracy can be judged by analysis of results obtained from known samples. The non-radionuclide contract laboratories analyze commercial reference samples every quarter at EPD/EMS' request. The results of these analyses are presented in the EPD/EMS groundwater monitoring quarterly reports. The primary laboratories also seek or maintain state certification by participating periodically in performance studies; reference samples and analysis of results are provided by EPA. Results of these studies also are published in the EPD/EMS quarterly reports.

Analysis of blanks provides a tool for assessing the accuracy of both sampling and laboratory analysis. Results for all field blanks for the quarter can be found in the EPD/EMS quarterly reports. Any field or laboratory blanks that exceed established minimums are identified in the same reports, in tables associating them with groundwater samples analyzed in the same batches. In these regulatory reports, the modifier $V$ is assigned to every result that is run in the same batch as a laboratory blank with positive results for that analyte. A second $V$ qualifier is added if the analyte concentration is less than five times the concentration in the blank (or for common laboratory contaminants less than ten times). 
Surrogates, organic compounds similar in chemical behavior to the compounds of interest but not normally found in environmental samples, are used to monitor the effect of the matrix on the accuracy of analyses for organic parameters. For example, for analyses of volatile organics by EPA Method 8240 , three surrogate compounds are added to all samples and blanks in each analytical batch. In analyses of semivolatile organics, three acid compounds and three base/ neutral compounds are used. Two surrogates are used in organochlorine pesticides analyses. Percent recoveries for surrogate analyses are calculated by laboratory personnel, reported to EPD/EMS, reviewed, and entered into the database, but they are not published. If recoveries are not within specified limits, the laboratory is expected to reanalyze the samples or attach qualifiers to the data identifying the anomalous results.

Sample-specific accuracy for both organic and inorganic parameters can be assessed by examination of matrix spike/matrix spike duplicate results. A portion of the sample is analyzed unspiked to determine a baseline set of values. A second portion of the sample is spiked with known concentrations of compounds appropriate to the analyses being performed, typically five volatile organic compounds for volatile organics analyses, eleven semivolatile compounds for semivolatiles, six pesticide compounds for pesticides, all metals for metals analyses by SW-846 methods (EPA, 1986), and a known quantity of cyanide for cyanide analysis. The percentage of the spike compound that is recovered (i.e., measured in excess of the value obtained for the unspiked sample) is a direct measure of analytical accuracy. EPA requires matrix spike/matrix spike duplicates to be run at least once per 20 samples of similar matrix.

Matrix spike/matrix spike duplicate results are reported to EPD/EMS but are not published. For organic compounds, according to EPA guidelines, no action is taken on the basis of matrix spikel matrix spike duplicate data alone (i.e., no result modifiers are assigned solely on the basis of matrix spike results); however, the results can indicate if a laboratory is having a systematic problem in the analysis of one or more analytes.

In the case of inorganic compounds, such as metals, the matrix spike sample analysis provides information about the effect of each sample matrix on the digestion and measurement methodology. Data qualifiers assigned by the laboratories on the basis of the percentage of spike recovery are reported in the EPD/EMS results tables.

\section{Assessment of Precision}

Precision of the analyses, or agreement of a set of replicate results among themselves, is assessed through the use of duplicates initiated by the laboratory and blind replicates provided by EPD/EMS. The results of duplicate and replicate analyses are presented in results tables that report only one quarter of data, usually during first, second, and third quarters. Duplicate and replicate results are not presented in results tables that report more than one quarter of data, generally provided in fourth quarter reports. In this case, the results tables instead present only the highest result for each analyte for each quarter of the year.

The laboratories assess precision by calculating the relative percent difference (RPD) for each pair of laboratory-initiated duplicate results. The contract laboratories use a data qualifier for metals analyses when the RPD for laboratory duplicates is greater than 20 percent. This qualifier is published in the EPD/EMS quarterly reports.

Additional statistical comparisons of laboratory duplicate and blind replicate results, both intraand interlaboratory, are presented in the EPD/EMS quarterly reports. The calculation used for these reports is the mean relative difference (MRD), which is similar to EPA's RPD except that the MRD is the average of all the RPD values from one laboratory for each compound 
(intralaboratory MRD) or all the RPD values from all laboratories for each compound (interlaboratory MRD) during one quarter. Because detection limits may vary among samples, the MRD requires calculation of a reference detection limit, which is the detection limit at the 90th percentile of the array of limits in the population of all duplicate and replicate analyses for a given analyte during a particular quarter. The MRD is not method-specific.

\section{Method-Specific Accuracy and Precision}

The contract laboratories' EPA-approved laboratory procedures include QAVC requirements as an integral part of the methods. Thus, knowledge of the method used in obtaining data is an important component of determining data usability. EPA has conducted extensive research and development on the methods approved for the analysis of water and wastewater. Information on the accuracy and precision of a method is available from EPA publications, as is full information on required $Q A / Q C$ procedures. A listing of the methods used by the primary laboratories during fourth quarter 1993 is given below along with the source for the method description. Many, if not all, of these sources include presentations of representative accuracy and precision results. The quarterly SRS Groundwater Monitoring Program report provides the methods used by the laboratories during each quarter.

\section{Methods Used by the Contract Laboratories}

\begin{tabular}{|c|c|c|}
\hline Method & Used to Analyze & Source \\
\hline EPA120.1 & Specific conductance & EPA EMSL, 1983 \\
\hline EPA150.1 & $\mathrm{pH}$ & EPA EMSL, 1983 \\
\hline EPA160.1 & Total dissolved solids & EPA EMSL, 1983 \\
\hline EPA160.2 & Total dissolved solids, total suspended solids & EPA EMSL, 1983 \\
\hline EPA180.1 & Turbidity & EPA EMSL, 1983 \\
\hline EPA200.7 & Metals & EPA EMSL, 1983 \\
\hline EPA204.2 & Antimony & EPA EMSL, 1983 \\
\hline EPA206.2 & Arsenic & EPA EMSL, 1983 \\
\hline EPA239.2 & Lead & EPA EMSL, 1983 \\
\hline EPA245.1 & Mercury & EPA EMSL, 1983 \\
\hline EPA270.2 & Selenium & EPA EMSL, 1983 \\
\hline EPA279.2 & Thallium & EPA EMSL, 1983 \\
\hline EPA300.0 & Chloride, nitrite, sulfate & EPA EMSL, 1991 \\
\hline EPA310.1 & Alkalinity & EPA EMSL, 1983 \\
\hline EPA325.2 & Chloride & EPA EMSL, 1983 \\
\hline EPA335.3 & Cyanide & EPA EMSL, 1983 \\
\hline EPA340.2 & Fluoride & EPA EMSL, 1983 \\
\hline EPA353.1 & Nitrogen, nitrate-nitrite & EPA EMSL, 1983 \\
\hline EPA353.2 & Nitrogen, nitrate, nitrite, or combined & EPA EMSL, 1983 \\
\hline EPA365.1 & Phosphorus, all forms (reported as total phosphates) & EPA EMSL, 1983 \\
\hline EPA365.2 & Phosphorus, all forms (reported as total phosphates) & EPA EMSL, 1983 \\
\hline EPA376.2 & Sulfide & EPA EMSL, 1983 \\
\hline EPA413.1 & Oil \& grease & EPA EMSL, 1983 \\
\hline EPA415.1 & $\begin{array}{l}\text { Dissolved organic carbon, total inorganic carbon, total organic } \\
\text { carbon }\end{array}$ & EPA EMSL, 1983 \\
\hline EPA418.1 & Total petroleum hydrocarbons & EPA EMSL, 1983 \\
\hline EPA420.2 & Phenols & EPA EMSL, 1983 \\
\hline EPA900.0 & Gross alpha, nonvolatile beta & EPA EMSL, 1980 \\
\hline EPA900.1 & Total alpha-emitting radium & EPA EMSL, 1980 \\
\hline EPA906.0 & Tritium & EPA EMSL, 1980 \\
\hline EPA6010 & Metals & EPA, 1986 \\
\hline EPA7041 & Antimony & EPA, 1986 \\
\hline
\end{tabular}


Method

EPA7060

EPA7421

EPA7470

EPA7740

EPA7841

EPA8010

EPA8080

EPA8150

EPA8240

EPA8270

EPA8280

EPA9012

EPA9020

EPA9020A

EPA9030

EPA9060
Used to Analyze

Arsenic

Lead

Mercury

Selenium

Thallium

Chlorinated volatile organics

Organochlorine pesticides and PCBs

Chlorinated herbicides

GCMS volatiles

GCMS semivolatiles

Dioxins and furans

Cyanide

Total organic halogens

Total organic halogens

Sulfide

Dissolved organic carbon, total inorganic carbon, total organic carbon
Source

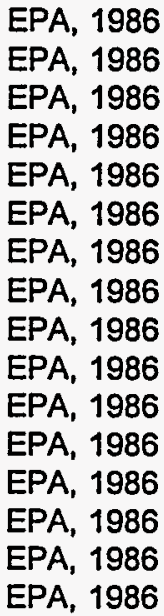

EPA, 1986

EPA, 1986

EPA, 1986

EPA, 1986

EPA, 1986

EPA 1986

EPA, 1986

EPA, 1986

EPA 1986

EPA, 1986

An example of available method-specific QA/QC information is that for the analysis of metals by EPA Method 6010/200.7 (EPA, 1986/EPA EMSL, 1983). The primary laboratories, General Engineering Laboratories (GE) and Roy F. Weston, Inc. (Weston), use this inductively coupled plasma (ICP) atomic emission spectrometric method.

The following precision and accuracy data are based on the experience of seven laboratories that applied the ICP technique to acid-distilled water matrices that had been spiked with various metal concentrates. (Note: Not all seven laboratories analyzed all 14 elements.) The references give results for samples having three concentration ranges; the results here are for samples having the lowest values, similar to actual groundwater results for the Savannah River Site.

\section{ICP Precision and Accuracy Data}

\begin{tabular}{|c|c|c|c|}
\hline Element & True value $(\mu \mathrm{g} / \mathrm{L})$ & $\begin{array}{l}\text { Mean reported } \\
\text { value }(\mu \mathrm{g} / \mathrm{L})\end{array}$ & $\begin{array}{l}\text { Mean percent } \\
\text { RSD }^{\mathrm{a}}\end{array}$ \\
\hline Aluminum & 60 & 62 & 33 \\
\hline Arsenic & 22 & 19 & 23 \\
\hline Beryllium & 20 & 20 & 9.8 \\
\hline Cadmium & 2.5 & 2.9 & 16 \\
\hline Chromium & 10 & 10 & 18 \\
\hline Cobalt & 20 & 20 & 4.1 \\
\hline Copper & 11 & 11 & 40 \\
\hline Iron & 20 & 19 & 15 \\
\hline Lead & 24 & 30 & 32 \\
\hline Manganese & 15 & 15 & 6.7 \\
\hline Nickel & 30 & 28 & 11 \\
\hline Selenium & 6 & 8.5 & 42 \\
\hline Vanadium & 70 & 69 & 2.9 \\
\hline Zinc & 16 & 19 & 45 \\
\hline
\end{tabular}

a Relative standard deviation. In EPA (1986), the column heading is Mean Standard Deviation (\%). 
As another example, EPA Method 601/8010 (EPA, 1991/EPA, 1986) is used by both GE and Weston for analyses of halogenated volatile organics. In the presentation of the method in both references, the following table gives method-specific accuracy and precision as functions of concentration. Contract laboratories are expected to achieve or at least approach these limits.

\section{Accuracy and Precision as Functions of Concentration for EPA Method 601/8010}

\section{Parameter}

Bromodichloromethane
Bromoform
Bromomethane
Carbon tetrachloride
Chlorobenzene
Chloroethane
2-Chloroethyl vinyl ether
Chloroform
Chloromethane
Dibromochloromethane
1,2-Dichlorobenzene
1,3-Dichlorobenzene
1,4-Dichlorobenzene
1,1-Dichloroethane
1,2-Dichloroethane
1,1-Dichloroethylene
trans-1,2-Dichloroethylene
Dichloromethane
1,2-Dichloropropane
cis-1,3-Dichloropropene
trans-1,3-Dichloropropene
1,1,2,2-Tetrachloroethane
Tetrachloroethylene
1,1,1-Trichloroethane
1,1,2-Trichloroethane
Trichloroethylene
Trichlorofluoromethane
Vinyl chloride

\begin{tabular}{|c|c|c|}
\hline $\begin{array}{l}\text { Accuracy as } \\
\text { recovery, } X^{\prime a}(\mu \mathrm{g} / \mathrm{L})\end{array}$ & $\begin{array}{l}\text { Single analyst } \\
\text { precision }(\mu \mathrm{g} / \mathrm{L})^{b}\end{array}$ & $\begin{array}{l}\text { Overall } \\
\text { precision }(\mu \mathrm{g} / \mathrm{L})^{c}\end{array}$ \\
\hline $1.12 C-1.02^{d}$ & $0.11 \bar{X}+0.04^{e}$ & $0.20 \bar{X}+1.00$ \\
\hline $0.96 C-2.05$ & $0.12 \bar{X}+0.58$ & $0.21 \bar{X}+2.41$ \\
\hline $0.76 C-1.27$ & $0.28 \bar{X}+0.27$ & $0.36 \bar{X}+0.94$ \\
\hline $0.98 C-1.04$ & $0.15 \bar{X}+0.38$ & $0.20 \overline{\mathrm{X}}+0.39$ \\
\hline $1.00 C-1.23$ & $0.15 \bar{X}-0.02$ & $0.18 \bar{X}+1.21$ \\
\hline $0.99 C-1.53$ & $0.14 \bar{X}-0.13$ & $0.17 \bar{X}+0.63$ \\
\hline $1.00 \mathrm{C}$ & $0.20 \bar{X}$ & $0.35 \bar{X}$ \\
\hline $0.93 C-0.39$ & $0.13 \bar{X}+0.15$ & $0.19 \bar{X}-0.02$ \\
\hline $0.77 C+0.18$ & $0.28 \bar{X}-0.31$ & $0.52 \bar{X}+1.31$ \\
\hline $0.94 C+2.72$ & $0.11 \bar{X}+1.10$ & $0.24 \bar{X}+1.68$ \\
\hline $0.93 C+1.70$ & $0.20 \bar{X}+0.97$ & $0.13 \bar{X}+6.13$ \\
\hline $0.95 C+0.43$ & $0.14 \bar{X}+2.33$ & $0.26 \bar{X}+2.34$ \\
\hline $0.93 C-0.09$ & $0.15 \bar{X}+0.29$ & $0.20 \bar{X}+0.41$ \\
\hline $0.95 C-1.08$ & $0.09 \bar{X}+0.17$ & $0.14 \bar{X}+0.94$ \\
\hline $1.04 C-1.06$ & $0.11 \bar{X}+0.70$ & $0.15 \bar{X}+0.94$ \\
\hline $0.98 C-0.87$ & $0.21 \bar{X}-0.23$ & $0.29 \bar{X}-0.40$ \\
\hline $0.97 C-0.16$ & $0.11 \bar{X}+1.46$ & $0.17 \bar{X}+1.46$ \\
\hline $0.91 C-0.93$ & $0.11 \bar{X}+0.33$ & $0.21 \bar{X}+1.43$ \\
\hline $1.00 \mathrm{C}$ & $0.13 \bar{x}$ & $0.23 \bar{X}$ \\
\hline $1.00 \mathrm{C}$ & $0.18 \bar{X}$ & $0.32 \bar{X}$ \\
\hline $1.00 \mathrm{C}$ & $0.18 \bar{X}$ & $0.32 \bar{X}$ \\
\hline $0.95 C+0.19$ & $0.14 \bar{X}+2.41$ & $0.23 \bar{X}+2.79$ \\
\hline $0.94 C+0.06$ & $0.14 \bar{X}+0.38$ & $0.18 \bar{X}+2.21$ \\
\hline $0.90 C-0.16$ & $0.15 \bar{X}+0.04$ & $0.20 \bar{X}+0.37$ \\
\hline $0.86 C+0.30$ & $0.13 \bar{X}-0.14$ & $0.19 \bar{X}+0.67$ \\
\hline $0.87 C+0.48$ & $0.13 \bar{X}-0.03$ & $0.23 \bar{X}+0.30$ \\
\hline $0.89 C-0.07$ & $0.15 \bar{X}+0.67$ & $0.26 \bar{X}+0.91$ \\
\hline $0.97 C-0.36$ & $0.13 \bar{X}+0.65$ & $0.27 \bar{X}+0.40$ \\
\hline
\end{tabular}

\footnotetext{
a $X^{\prime}=$ expected recovery for one or more measurements of a sample containing a concentration of $C$, in $\mu \mathrm{g} / \mathrm{L}$.

b Expected single analyst standard deviation of measurements.

c Expected interlaboratory standard deviation of measurements.

d $C=$ true value for the concentration, in $\mu \mathrm{g} / \mathrm{L}$.

e $\bar{X}=$ average recovery found for measurements of samples containing a concentration of $C$, in $\mu \mathrm{g} / \mathrm{L}$.

$f$ Estimates based on performance of a single laboratory.
} 


\section{References Cited}

EPA (U.S. Environmental Protection Agency), 1986. Test Nethods for Evaluating Solid Waste (SW-846), Volumes IA-IC. Washington, DC.

EPA (U.S. Environmental Protection Agency), 1991. Guidelines Establishing Test Procedures for the Analysis of Pollutants, Code of Federal Regulations, Title 40, Part 136, Appendix A. Washington, DC.

EPA EMSL (U.S. Environmental Protection Agency, Environınental Monitoring and Systems Laboratory), 1980. Prescribed Procedures for Measurement of Radioactivity in Drinking Water, EPA-600/4-80-032. Cincinnati, OH.

EPA EMSL (U.S. Environmental Protection Agency, Environmental Monitoring and Systems Laboratory), 1983. Methods for Chemical Analysis of Water and Wastes. Cincinnati, OH.

EPA EMSL (U.S. Environmental Protection Agency, Environınental Monitoring and Systems Laboratory), 1991. Test Method, The Determination of Inorganic Anions in Water by lon Chromatography-Method 300.0. Cincinnati, $\mathrm{OH}$. 